\title{
Three-dimensional simulations of non-stationary accretion by remnant black holes of compact object mergers
}

\author{
S. Setiawan ${ }^{1}$, M. Ruffert ${ }^{1}$, and H.-Th. Janka ${ }^{2}$ \\ 1 School of Mathematics, University of Edinburgh, Edinburgh EH9 3JZ, Scotland, UK \\ e-mail: [S.Setiawan; M.Ruffert]@ed.ac .uk \\ 2 Max-Planck-Institut für Astrophysik, Postfach 1317, 85741 Garching, Germany \\ e-mail: thj@mpa-garching.mpg.de
}

Received 12 September 2005 / Accepted 21 July 2006

ABSTRACT

\begin{abstract}
By means of three-dimensional hydrodynamic simulations with an Eulerian PPM code we investigate the time-dependent evolution and properties of accretion tori around nonrotating and rotating stellar-mass black holes, using a pseudo-Newtonian (Paczyński \& Wiita or Artemova-Björnsson-Novikov) potential to approximate the effects of general relativity. The simulations are performed with three nested Cartesian grids to ensure sufficient resolution near the central black hole on the one hand and a large computational volume on the other. The black hole and torus are considered as the remnant of a binary neutron star or neutron-star black-hole merger. Referring to results from previous hydrodynamical simulations of such events, we assume the initial configurations to consist of a black hole with a mass of about $4 M_{\odot}$ girded by a toroidal accretion disk with a mass in the range from about $0.01 M_{\odot}$ to $0.2 M_{\odot}$. We simulate the torus evolution without and with physical shear viscosity, employing a simple $\alpha$-model for the gas viscosity. As in our previous work on merging neutron star binaries and neutron star/black hole binaries, we use the equation of state of Lattimer and Swesty. The energy loss and lepton number change due to neutrino emission from the hot torus are treated by a neutrino-trapping scheme. The energy deposition by neutrino-antineutrino annihilation around the disk is evaluated in a post-processing step. The timedependent efficiency of converting gravitational energy to neutrinos, expressed by the ratio of neutrino luminosity to accretion rate of rest-mass energy, can reach maximum values of up to about $10 \%$. The corresponding efficiency of converting neutrino energy into a pair-photon fireball by neutrino annihilation peaks at values of several percent. Interestingly, we find that the rate of neutrinoantineutrino annihilation decays with time much less steeply than the total neutrino luminosity does with the decreasing gas mass of the torus, because the ongoing protonization of the initially neutron-rich disk matter leads to a rather stable product of neutrino and antineutrino luminosities. The neutrino luminosity and total energy release of the torus increase steeply with higher viscosity, larger torus mass, and larger black hole spin in corotation with the disk, in particular when the spin parameter is $a \gtrsim 0.8$. The latter dependence is moderated in case of a high disk viscosity. For rotation rates as expected for post-merger black holes $(a \gtrsim 0.5)$ and reasonable values of the alpha viscosity of the torus $(\alpha \sim 0.1)$, torus masses in the investigated range can release sufficient energy in neutrinos to account for the energetics of the well-localized short gamma-ray bursts recently detected by Hete and Swift, if collimation of the ultrarelativistic outflows into about $1 \%$ of the sky is invoked, as predicted by recent hydrodynamic jet simulations.
\end{abstract}

Key words. accretion, accretion disks - hydrodynamics - elementary particles - gamma rays: bursts - stars: neutron

\section{Introduction}

Merging double neutron stars or neutron-star black-hole binaries have long been discussed as possible sources of cosmic gamma-ray bursts (GRBs) (e.g., Blinnikov et al. 1984; Paczyński 1986; Goodman 1986; Goodman et al. 1987; Eichler et al. 1989; Paczyński 1991; Narayan et al. 1992; Mészáros \& Rees 1993; Woosley 1993a; Jaroszyński 1993, 1996; Mochkovitch et al. 1993, 1995; Thompson 1994; Witt et al. 1994; Janka \& Ruffert 1996; Mészáros \& Rees 1997; Popham et al. 1999; Mészáros et al. 1999). After the discovery that long GRBs are linked to massive star explosions, which confirmed predictions by Woosley (1993a) and MacFadyen \& Woosley (1999), it is in particular the class of short, hard GRBs with durations of less than about 2 seconds, which is thought to originate from colliding compact objects in binary systems. Recent observations of well-localized short GRBs (Gehrels et al. 2005; Hjorth et al. 2005; Berger et al. 2005) seem to support this hypothesis, because in agreement with expectations short GRBs are found in galaxies without strong star-formation activity.
In the most popular scenario, GRBs have in common that they signal the birth of stellar-mass black holes and the associated huge energy release when the growing black hole accretes matter at enormous rates between fractions of a solar mass per second up to many solar masses per second (Woosley 1993a; Popham et al. 1999; MacFadyen \& Woosley 1999). The huge amount of gravitational binding energy released during the accretion process of up to several solar masses of gas into the black hole could explain the energetics of even the most distant cosmological gamma-ray bursts (e.g., GRB981214, see Kulkarni et al. 1998). Moreover, the compactness of the stellar-mass black hole could naturally produce the rapid variability on time scales of milliseconds observed in many bursts. For these reasons, massive accretion disks or thick accretion tori around stellar-mass black holes are considered as favourable model for the central engines of the cosmological GRBs.

In this scenario the energy of the ultra-relativistically expanding fireball or jet, can be provided by the annihilation of neutrino-antineutrino pairs (Paczyński 1991; Mészáros \& Rees 1997; Woosley 1993b; Jaroszyński 1993, 1996; 
Table 1. Parameters of the torus evolution models. The 3D simulations were performed with three levels of nested Cartesian grids with the number of grid cells per level and spatial direction given in column "zone". $\Delta t_{\text {cal }}$ is the time interval over which the model was evolved. All models started with a black hole mass of $M_{\mathrm{BH}}^{\mathrm{i}}=4.017 M_{\odot}$. The initial torus masses are given by $M_{\mathrm{d}}^{\mathrm{i}}, 0 \leq a \leq 1$ is the initial value of the dimensionless BH spin parameter, "pro" means that torus and BH were chosen to rotate in the same direction, "ret" means opposite directions of rotation, and $\alpha$ denotes the dimensionless disk viscosity parameter.

\begin{tabular}{lrrcccc}
\hline \hline Model & $\begin{array}{r}\text { Zone } \\
\end{array}$ & $\begin{array}{r}\Delta t_{\text {cal }} \\
\mathrm{ms}\end{array}$ & $\begin{array}{c}M_{\mathrm{d}}^{\mathrm{i}} \\
M_{\odot}\end{array}$ & $\begin{array}{c}\text { BH spin } \\
a\end{array}$ & Direction & $\begin{array}{c}\text { Visc } \\
\alpha\end{array}$ \\
\hline r00-32 & 32 & 70.0 & 0.0478 & 0.0 & - & 0.0 \\
al1-32 & 32 & 70.0 & 0.0478 & 0.0 & - & 0.001 \\
al2-32 & 32 & 70.0 & 0.0478 & 0.0 & - & 0.004 \\
al3-32 & 32 & 70.0 & 0.0478 & 0.0 & - & 0.01 \\
al4-32 & 32 & 70.0 & 0.0478 & 0.0 & - & 0.1 \\
ro1-32 & 32 & 70.0 & 0.0478 & 0.3 & pro & 0.0 \\
ro2-32 & 32 & 70.0 & 0.0478 & 0.6 & pro & 0.0 \\
ro3-32 & 32 & 70.0 & 0.0478 & 0.8 & pro & 0.0 \\
ro4-32 & 32 & 70.0 & 0.0478 & 0.3 & ret & 0.0 \\
ro5-32 & 32 & 70.0 & 0.0478 & 0.6 & ret & 0.0 \\
ir1-32 & 32 & 70.0 & 0.0120 & 0.0 & - & 0.0 \\
ir2-32 & 32 & 70.0 & 0.0239 & 0.0 & - & 0.0 \\
ir3-32 & 32 & 70.0 & 0.0956 & 0.0 & - & 0.0 \\
ir4-32 & 32 & 70.0 & 0.1912 & 0.0 & - & 0.0 \\
r00-64 & 64 & 40.0 & 0.0478 & 0.0 & - & 0.0 \\
a13-64 & 64 & 40.0 & 0.0478 & 0.0 & - & 0.01 \\
al4-64 & 64 & 40.0 & 0.0478 & 0.0 & - & 0.1 \\
ri4-64 & 64 & 40.0 & 0.1912 & 0.6 & pro & 0.0 \\
ro2-64 & 64 & 40.0 & 0.0478 & 0.6 & pro & 0.0 \\
ro5-64 & 64 & 40.0 & 0.0478 & 0.6 & ret & 0.0 \\
ir1-64 & 64 & 40.0 & 0.0120 & 0.0 & - & 0.0 \\
ir4-64 & 64 & 40.0 & 0.1912 & 0.0 & - & 0.0 \\
ir5-64 & 64 & 40.0 & 0.1912 & 0.0 & - & 0.1 \\
ar1-64 & 64 & 40.0 & 0.0478 & 0.6 & pro & 0.1 \\
ar2-64 & 64 & 40.0 & 0.1912 & 0.6 & pro & 0.1 \\
\hline
\end{tabular}

Mochkovitch et al. 1993, 1995) or possibly by magnetohydrodynamical processes (Blandford \& Znajek 1977; Mészáros \& Rees 1992; Popham et al. 1999; Li 2000; Lee et al. 2000; see also Livio et al. 1999; Rosswog \& Ramirez-Ruiz 2002; Rosswog et al. 2003). In the former case, the gravitational binding energy of accreted disk matter is tapped, in the latter case (also) the rotational energy of the central black hole $(\mathrm{BH})$ could be converted into kinetic energy of the outflow.

In a series of previous papers (Ruffert et al. 1996, 1997; Ruffert \& Janka 1999, 2001) it has been shown that the neutrino emission associated with the dynamical phase of the merging or collision of two neutron stars is not sufficiently powerful and too short to provide the energy for gamma-ray bursts by neutrinoantineutrino annihilation. After a transient phase of gravitational stability, the compact massive remnant of the neutron star merger may collapse to a black hole and some matter may remain in a hot toroidal accretion disk around the black hole, which continues to radiate neutrinos with high luminosities (for recent discussions and model calculations, see, e.g., Popham et al. 1999; Rosswog et al. 1999, 2000; Ruffert \& Janka 1999; Oechslin et al. 2004; Morrison et al. 2004; Oechslin \& Janka 2006).

Newtonian hydrodynamic simulations including a physical equation of state (Ruffert et al. 1996; Ruffert \& Janka 1999) indicate that about $0.1 M_{\odot}$ of matter might obtain enough angular momentum during the merging of the neutron stars to avoid immediate accretion by the black hole. These numbers have recently been confirmed by simulations including a treatment of general relativity (Oechslin \& Janka 2006). The presence of large angular momentum allows the matter to form and sustain toroidal disk around the black hole for a period of time depending on the viscous transport of angular momentum in the disk. Therefore there could be enough time for this material to radiate away in neutrinos a fair fraction of its gravitational binding energy. A similar situation could result from the merging of a neutron star with a black hole (Lee \& Kluźniak 1995, 1999; Kluźniak \& Lee 1998; Janka et al. 1999; Rosswog et al. 2004).

Many questions remain to be explored by quantitative calculations of the formation, evolution, and energy release of accreting stellar-mass black holes as possible central engines of GRBs. The results of the three-dimensional (3D) hydrodynamic simulations presented in this paper aim at studying the time-dependent evolution of such black holes girded by accretion tori that were formed from the decompressed matter of a neutron star, which was disrupted in a compact binary merger. Such tori are generated by a violent dynamical process in the immediate vicinity of the black hole. The assumption of stationary accretion is therefore valid only approximately and only after a longer phase of adjustment and relaxation of the matter girding the black hole. This relaxation can proceed over a fair fraction of the whole period of massive accretion and requires time-dependent modelling. Therefore our simulations intend to contribute to answering the following questions: how much mass remains in the accretion torus for longer than dynamical time scales? What is the mass accretion rate of the black hole as a function of time? What are the properties of the accretion torus, its density, temperature, neutron-to-proton ratio, neutrino luminosity as functions of time? How do they depend on torus mass, gas viscosity, and black hole rotation? How efficient is neutrino-antineutrino annihilation in depositing energy in the surrounding of the black-hole torus system in dependence of these parameters? What are the implications for powering ultrarelativistic outflow and gammaray bursts by neutrino-antineutrino annihilation?

Our 3D models of the time-dependent, non-stationary, evolution of the black-hole torus systems include a detailed treatment of the equation of state and neutrino physics. They thus replace approximations of previous work as made in the analytic studies of Popham et al. (1999) and Di Matteo et al. (2002) and in the numerical 2D models of Lee \& Ramirez-Ruiz (2002). These latter simulations with azimuthal symmetry employed a simple ideal gas equation state and made the assumption that all the dissipated energy is radiated away in neutrinos. Lee et al. (2004, 2005a,b), used a more realistic equation of state and took into account neutrino energy loss and opacity effects, but with a simplified model for changes lepton number $\left(Y_{\mathrm{e}}\right)$, considered only Newtonian gravity and nonrotating black holes, and did not evaluate their 2D hydrodynamic models for the energy deposition by neutrino-antineutrino annihilation.

The paper is organized as follows. In Sect. 2 we will introduce the numerical tools used for our simulations, outline the initial conditions, and give an overview over the different investigated cases. Section 3 will present the results of our simulations and discuss their implications. The summary of our main findings will be given in Sect. 5, and concluding remarks and an outlook will follow in Sect. 6.

\section{Numerical models}

\subsection{Dynamics}

The three-dimensional computations of black-hole accretiondisk dynamics in this paper were performed with a Newtonian 


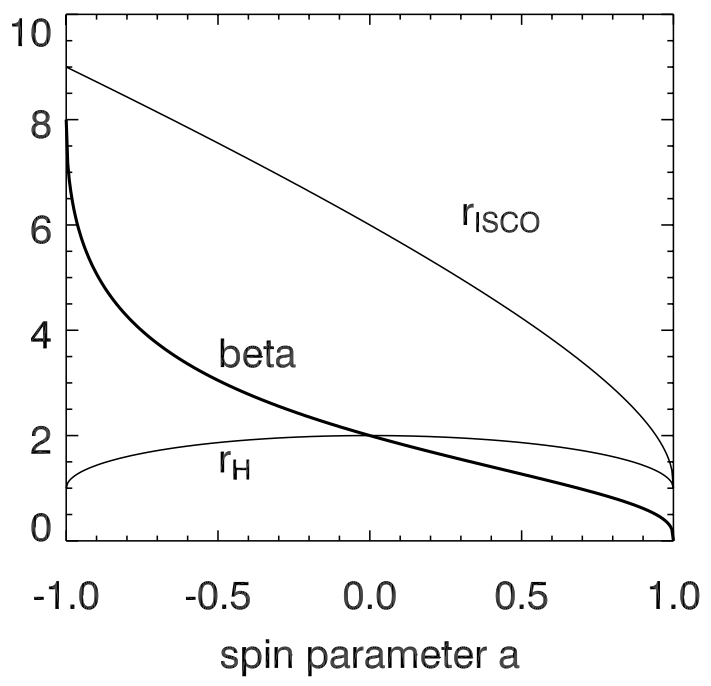

Fig. 1. The black hole event horizon $r_{\mathrm{H}}$, the innermost stable circular orbit $r_{\mathrm{ISCO}}$, and $\beta$ as defined in Eq. (2), as functions of the black hole spin parameter $a$. Both $r_{\mathrm{H}}$ and $r_{\mathrm{ISCO}}$ are given in units of $G M_{\mathrm{BH}} / c^{2}=0.5 R_{\mathrm{s}}$.

hydrodynamics code based on the Piecewise Parabolic Method (PPM) of Colella \& Woodward (1984) with three levels of nested grids (Ruffert 1992) to ensure both sufficient resolution near the central black hole and a large computational volume. A higher grid level has twice the zone size of the level below. With an equatorial length and width of the total computational volume of $500 \mathrm{~km}$, the smallest zones have a side length of $1.95 \mathrm{~km}$ for 64 zones per grid and dimension ("64-resolution") and $3.90 \mathrm{~km}$ for 32 zones on each grid level ("32-resolution").

The "black hole" with mass $M_{\mathrm{BH}}$ is treated as a gravitational centre surrounded by a vacuum sphere on the computational grid. Its gravitational potential $\Phi_{\mathrm{BH}}$ as a function of radius $r$ is calculated from

$\frac{\mathrm{d} \Phi_{\mathrm{BH}}}{\mathrm{d} r}=-\frac{G M_{\mathrm{BH}}}{r^{2-\beta}\left(r-r_{\mathrm{H}}\right)^{\beta}}$,

where $r_{\mathrm{H}}$ is the black hole event horizon, and $\beta$ is a constant for a given value of the black hole spin parameter $a$. It is defined by

$\beta=\frac{r_{\mathrm{ISCO}}}{r_{\mathrm{H}}}-1$.

Here the radius of the innermost stable circular orbit (ISCO), $r_{\text {ISCO }}$, is the same as $r_{\text {in }}$ in Artemova et al. (1996). Both $r_{\mathrm{H}}$ and $r_{\text {ISCO depend on } a \text { as shown in Fig. } 1 .}$

This potential reduces to the usual Paczyński-Wiita potential (Paczyński \& Wiita 1980) when the black hole spin parameter $a$ is zero. It allows us to mimic important general relativistic effects, in particular to reproduce the existence of a last stable circular orbit.

The local Keplerian velocity for the Artemova-BjörnssonNovikov potential is

$v_{\text {Kepler }}(r)=\sqrt{\frac{G M(r) r^{\beta-1}}{\left(r-r_{\mathrm{H}}\right)^{\beta}}}$,

with $M(r)$ being the mass enclosed by the sphere of radius $r$ and $\beta$ being given by Eq. (2). This becomes the Keplerian velocity of the Paczyński-Wiita potential when the black hole spin parameter $a$ is zero and thus $\beta=2$.
The radius of the vacuum sphere mimicking the black hole is chosen to be the arithmetic mean of event horizon and innermost stable circular orbit. The outflow boundary condition allows mass and angular momentum (and energy) to leave the grid. The mass and the spin of the black hole are accordingly updated during the simulations. The corresponding changes of the black hole spin are, however, minor (cf. Setiawan et al. 2004) because the accreted mass is small compared to the initial mass of the black hole in all cases.

So the only GR effect being modeled is the reduction in radius of the ISCO. Explicit frame dragging is omitted, as well as the gravitational lensing effect which might capture some of the neutrinos emitted in this region. A detailed discussion of the lensing effect can be found in Sect. 7.2 of Ruffert \& Janka (1999), where we find a moderate decrease of the annihilation energy by $10 \%-30 \%$.

\subsection{Viscosity}

In our simulations we compare the evolution of the disk with and without shear viscosity. The viscosity source terms for energy, momentum components, and entropy equations can be expressed, respectively, as follows

$$
\begin{aligned}
& V_{\mathrm{E}}=\frac{\eta_{\mathrm{p}}}{2}\left(\frac{\partial v^{i}}{\partial x^{j}}+\frac{\partial v^{j}}{\partial x^{i}}-\frac{2}{3} \delta_{i j} \frac{\partial v^{k}}{\partial x^{k}}\right)^{2} \\
& V_{\mathrm{mom}}^{i}=\frac{\partial}{\partial x^{j}}\left[\eta_{\mathrm{p}}\left(\frac{\partial v^{i}}{\partial x^{j}}+\frac{\partial v^{j}}{\partial x^{i}}-\frac{2}{3} \delta_{i j} \frac{\partial v^{k}}{\partial x^{k}}\right)\right] \\
& V_{\mathrm{ent}}=\frac{1}{k_{\mathrm{B}} T}\left[\frac{\eta_{\mathrm{p}}}{2}\left(\frac{\partial v^{i}}{\partial x^{j}}+\frac{\partial v^{j}}{\partial x^{i}}-\frac{2}{3} \delta_{i j} \frac{\partial v^{k}}{\partial x^{k}}\right)^{2}\right],
\end{aligned}
$$

where $\eta_{\mathrm{p}}$ is the dynamical shear viscosity coefficient parameterized by the simple expression:

$\eta_{\mathrm{p}}=\alpha \rho c_{\mathrm{s}}^{2} / \Omega_{K}$

Here $\alpha$ is the standard dimensionless disk-viscosity parameter introduced by Shakura \& Sunyaev (1973), $\Omega_{\mathrm{K}}$ is the Keplerian angular velocity, and $c_{\mathrm{s}}=(\Gamma P / \rho)^{1 / 2}$ is the adiabatic sound speed with $\Gamma$ being the adiabatic index. The expressions of Eqs. (4) and (5) were added as source terms to the energy and momentum evolution equations of our PPM scheme.

We use the subscript $p$ for the shear viscosity that is assumed to result from physical processes in the disk (e.g. turbulence or magnetohydrodynamic processes, see Balbus \& Hawley 1998; Duez et al. 2004; Thompson et al. 2005) to distinguish it from the numerical viscosity which was discussed in Ruffert \& Janka (2001) and Ruffert \& Janka (1999). The approach used in this paper is similar to the disk formalism developed by Chen et al. (1995), Abramowicz et al. (1995), Lee \& Ramirez-Ruiz (2002), and McKinney \& Gammie (2002); see also Hawley \& Krolik (2000), Narayan et al. (2001), and Kohri \& Mineshige (2002).

The resolution dependent viscous dissipation due to numerical viscosity can be estimated to correspond to an $\alpha$ value of approximately 0.01 for our code and the chosen grid zoning (see Ruffert \& Janka 2001; and Janka et al. 1999).

\subsection{Thermodynamics}

The thermodynamics of the gas is described by using the equation of state (EoS) of Lattimer \& Swesty (1991). This allows 
us to follow the neutrino production and escape by a leakage and trapping scheme as detailed in previous papers (Ruffert et al. 1996, 1997; Ruffert \& Janka 1999, 2001) in order to calculate the energy and lepton number changes by neutrino losses. This approach is used in the present paper, because our main aim is the investigation of the relevance for gamma-ray burst scenarios, in particular for those where the neutrino emission has been suggested to provide the energy for the relativistic gammaray burst fireball via neutrino-antineutrino annihilation. Also the amount of mass ejection during the dynamical interaction and the properties of the ejected matter depend on the EoS.

The density is bounded from below in our simulations by the assumption of an environmental medium of about $10^{8} \mathrm{~g} \mathrm{~cm}^{-3}$ (determined by the minimum density of the employed equation of state table) and is more than two orders of magnitude below the average densities inside our simulated tori.

In addition to the standard equations of continuity and conservation of momentum and energy, we also evolve an entropy equation (more information can be found in Ruffert \& Janka (2001). The terms due to viscosity are given in Sect. 2.2. This allows us to obtain the temperature from both the energy and the entropy equation, allowing a cross-check. For the simulations of the torus evolution shown in this paper, the two temperatures are in fairly good overall agreement, so this double-check of the temperature is redundant in the context for the simulations presented in this paper. Local differences between the two temperatures remain local and are therefore of no major importance for the overall hydrodynamical results.

\subsection{Models and initial conditions}

Table 1 contains a list of computed models with their specific parameters, including the values of $\alpha$. We follow the non-stationary evolution of the toroidal disk around the central black hole on much longer time scale than the dynamical time scale of the merger of black hole/neutron star systems, considering the viscous transport of angular momentum which depends on the assumed shear viscosity of the disk which we parametrise in the models. Model "r00" defines a reference case without black hole rotation and without disk viscosity. The "al" models include disk viscosity but no black hole spin, models "ro" and "ri" consider rotating black holes but no disk viscosity, and models "ar" take into account non-zero values for the disk viscosity and BH spin. The names of the models also carry information about the number of zones of each grid level (32 or 64).

The data for our initial setups, which consist of a black hole and an axisymmetric, toroidal accretion disk with chosen masses, were taken from one of the final configurations obtained in the simulations described by Janka et al. (1999; see also Ruffert et al. 1996, 1997; and Ruffert \& Janka 2001, for more information). Density, temperature, and neutron-to-proton ratio of the matter, which is assumed to be in nuclear statistical equilibrium, were azimuthally averaged. Because we varied the disk mass by simply scaling the density, and moreover switched from the Newtonian potential of the merger runs to the pseudoNewtonian gravity in this work, the tori are initially slightly out of rotational equilibrium. Despite the artificial adjustment which happens during the first milliseconds of our simulations, we do not consider this as a major problem because the compact object merger as well as a subsequent collapse of the merger remnant to a black hole are violent processes, followed naturally by a relaxation phase of the mass left in a torus.

The simulations of the present work started with a black hole mass of $4.017 M_{\odot}$. This is the mass produced by the final
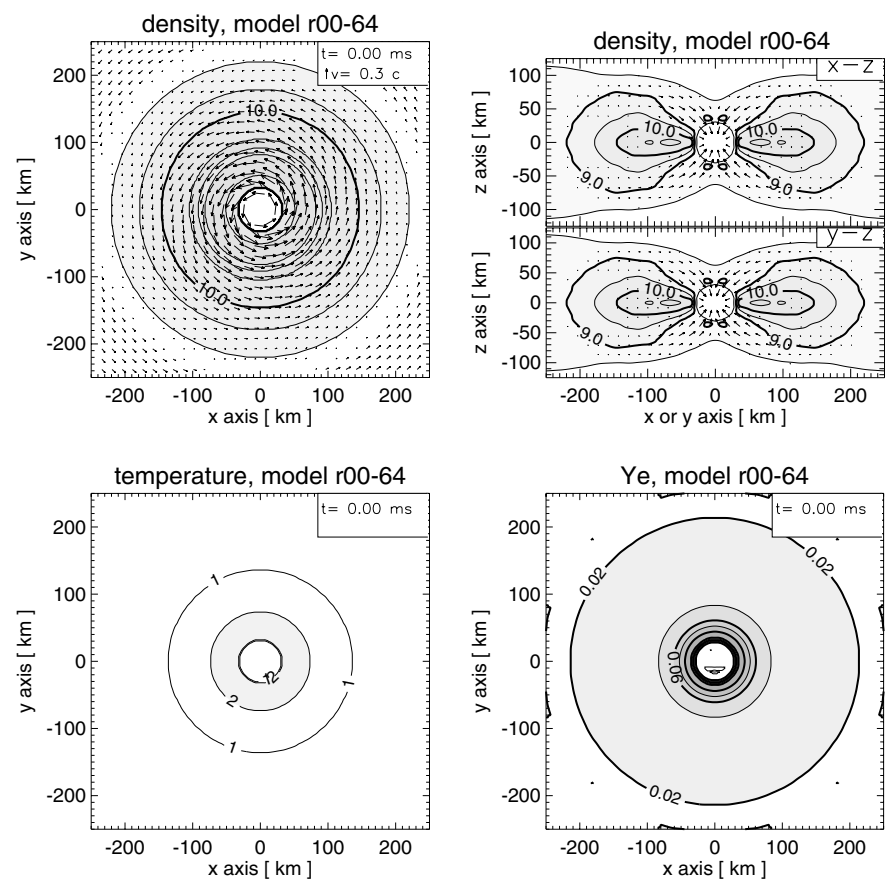

Fig. 2. Initial configuration at the start of the simulations for model r0064. The density is displayed in the orbital plane (left) and in two orthogonal planes perpendicular to the equator (right). It is given in $\mathrm{g} \mathrm{cm}^{-3}$ with contours spaced logarithmically in steps of 0.5 dex. The arrows in the density plots indicate the velocity field. The temperature and electron fraction $\left(Y_{\mathrm{e}}\right)$ are displayed in the orbital plane. The temperature is measured in $\mathrm{MeV}$, its contours are labelled with the corresponding values. The contours of electron fraction are spaced linearly in steps of 0.02 .

configurations of the models described above. We will use the model with a torus mass of $0.0478 M_{\odot}$, as "reference model". The mass was then also changed to values between $0.0120 M_{\odot}$ and $0.1912 M_{\odot}$ (see Table 1 ) by simply scaling the density distribution of the reference configuration with an appropriate factor. The initial conditions of temperature, density, and electron fraction (i.e., proton-to-baryon ratio) for the reference case are shown in Fig. 2.

The initial black hole spin parameter was varied between values of $0,0.3,0.6$ or 0.8 for 32 -resolution models and between 0 or 0.6 for 64-resolution models, respectively. We did not choose $a$ closer to unity because our code is not fully relativistic. When $a$ is near unity, the disk moves in so close to the event horizon that general relativistic effects are extremely strong and the fluid velocities approach the speed of light, thus making the approximative description of relativistic gravity and the nonrelativistic fluid dynamics of our code questionable.

\section{Results}

In this section we will discuss only the models calculated with a resolution of 64 zones on each level of the nested grid, so the specification "-64" in the model name, e.g. r00-64, will be mostly omitted. Comparing runs with 32 zones and 64 zones at the same time, one finds only relatively small differences of the model evolution and global disk properties, of which some are given in Table 2. The 3D simulations with 32 zones, however, could be performed for a time interval of $70 \mathrm{~ms}$, whereas computer resources did not allow us to follow the evolution of the better resolved models for more than $40 \mathrm{~ms}$. In Table 2 we also 
Table 2. Some results for the torus evolution models. The dynamical simulations were performed over a time interval of $\Delta t_{\text {cal }}$. All quantities refer to the conditions found at the end of the simulations. $M_{\mathrm{d}}$ is the mass of the gas of the disk, $T_{\max }$ is the maximum gas temperature in energy units, $L_{v_{\mathrm{e}}}$ denotes the electron neutrino luminosity near the end of the simulation, $L_{\bar{v}_{\mathrm{e}}}$ the corresponding electron antineutrino luminosity, and $L_{v_{x}}$ the luminosity of heavy-lepton neutrinos (summed for $v_{\mu}, \bar{v}_{\mu}, v_{\tau}$ or $\bar{v}_{\tau}$, which are all treated equally). The sum of all neutrino luminosities is given by $L_{v}$, and the mean energies of the different neutrino types by $\left\langle\epsilon_{v_{\mathrm{e}}}\right\rangle,\left\langle\epsilon_{\bar{v}_{\mathrm{e}}}\right\rangle$ and $\left\langle\epsilon_{v_{x}}\right\rangle$.

\begin{tabular}{|c|c|c|c|c|c|c|c|c|c|c|}
\hline Model & $\begin{array}{l}\Delta t_{\mathrm{cal}} \\
\mathrm{ms}\end{array}$ & $\begin{array}{c}M_{\mathrm{d}} \\
10^{-2} M_{\odot}\end{array}$ & $\begin{array}{l}T_{\max } \\
\mathrm{MeV}\end{array}$ & $\begin{array}{c}L_{v_{\mathrm{e}}} \\
10^{50} \frac{\mathrm{erg}}{\mathrm{s}}\end{array}$ & 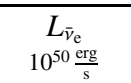 & 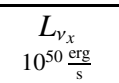 & $\begin{array}{c}L_{v} \\
10^{50 \frac{\mathrm{rg} g}{\mathrm{~s}}}\end{array}$ & $\begin{array}{l}\left\langle\epsilon_{\mathrm{ve}_{\mathrm{e}}}\right\rangle \\
\mathrm{MeV}\end{array}$ & $\begin{array}{l}\left\langle\epsilon_{\left.{\overline{v_{\mathrm{e}}}}\right\rangle}\right\rangle \\
\mathrm{MeV}\end{array}$ & $\begin{array}{l}\left\langle\epsilon_{v_{x}}\right\rangle \\
\mathrm{MeV}\end{array}$ \\
\hline $\mathrm{r} 00-32$ & 70.0 & 1.06 & 2.6 & 0.08 & 0.22 & 0.002 & 0.3 & 9.7 & 9.3 & 7.8 \\
\hline al1-32 & 70.0 & 1.14 & 3.3 & 0.2 & 0.6 & 0.01 & 0.8 & 12.5 & 12.0 & 10.0 \\
\hline al2-32 & 70.0 & 1.25 & 4.3 & 1.0 & 2.9 & 0.1 & 4.0 & 15.4 & 15.2 & 12.8 \\
\hline al3-32 & 70.0 & 1.43 & 5.0 & 1.4 & 3.9 & 0.2 & 5.5 & 17.5 & 17.2 & 14.7 \\
\hline al4-32 & 70.0 & 0.84 & 5.9 & 4.0 & 9.0 & 2.0 & 15.0 & 17.5 & 19.2 & 16.9 \\
\hline ro1-32 & 70.0 & 1.54 & 3.8 & 0.3 & 0.8 & 0.008 & 1.1 & 11.3 & 11.0 & 9.4 \\
\hline ro2-32 & 70.0 & 2.10 & 4.5 & 0.7 & 1.8 & 0.03 & 2.5 & 11.4 & 11.2 & 9.7 \\
\hline ro3-32 & 70.0 & 2.02 & 6.4 & 9.0 & 14.0 & 0.4 & 23.0 & 18.2 & 19.1 & 18.3 \\
\hline ro4-32 & 70.0 & 0.67 & 2.1 & 0.009 & 0.02 & 0.0006 & 0.03 & 8.5 & 8.3 & 6.9 \\
\hline ro5-32 & 70.0 & 0.56 & 1.8 & $<0.005$ & $<0.01$ & $<0.0002$ & $<0.015$ & 5.8 & 6.1 & 5.4 \\
\hline ir1-32 & 70.0 & 0.46 & 1.4 & $<0.0005$ & $<0.0005$ & $<0.0001$ & $<0.001$ & 5.1 & 5.6 & 4.7 \\
\hline ir $2-32$ & 70.0 & 0.57 & 1.9 & $<0.0025$ & $<0.0025$ & $<0.0005$ & $<0.005$ & 6.8 & 6.7 & 5.7 \\
\hline ir3-32 & 70.0 & 2.35 & 3.1 & 1.7 & 2.4 & 0.035 & 4.1 & 11.9 & 11.4 & 9.6 \\
\hline ir4-32 & 70.0 & 4.31 & 7.1 & 34.0 & 50.0 & 2.0 & 86.0 & 17.3 & 17.3 & 17.0 \\
\hline r00-64 & 40.0 & 2.22 & 4.2 & 0.6 & 1.2 & 0.01 & 1.8 & 12.0 & 11.6 & 10.2 \\
\hline al3-64 & 40.0 & 2.44 & 5.6 & 3.4 & 7.7 & 0.3 & 11.4 & 16.4 & 17.8 & 15.4 \\
\hline al4-64 & 40.0 & 1.76 & 8.7 & 18.0 & 55.0 & 7.0 & 80.0 & 21.3 & 22.8 & 20.1 \\
\hline ri4-64 & 40.0 & 12.22 & 7.0 & 26.0 & 43.0 & 1.0 & 70.0 & 14.5 & 16.5 & 17.9 \\
\hline ro2-64 & 40.0 & 3.10 & 4.6 & 1.6 & 1.8 & 0.02 & 3.4 & 12.9 & 11.7 & 11.7 \\
\hline ro5-64 & 40.0 & 1.13 & 3.9 & 0.3 & 0.7 & 0.01 & 1.0 & 12.2 & 11.8 & 9.8 \\
\hline ir1-64 & 40.0 & 0.71 & 2.4 & 0.07 & 0.03 & 0.001 & 0.1 & 9.4 & 8.4 & 7.0 \\
\hline ir4-64 & 40.0 & 7.87 & 6.2 & 7.2 & 15.0 & 0.2 & 22.4 & 13.8 & 12.8 & 14.9 \\
\hline ir5-64 & 40.0 & 8.77 & 10.4 & 150.0 & 350.0 & 110.0 & 610.0 & 20.7 & 20.9 & 26.7 \\
\hline $\operatorname{ar} 1-64$ & 40.0 & 2.34 & 6.6 & 25.0 & 70.0 & 15.0 & 110.0 & 21.5 & 22.7 & 20.3 \\
\hline ar2-64 & 40.0 & 10.44 & 9.5 & 120.0 & 240.0 & 80.0 & 440.0 & 19.4 & 19.7 & 25.2 \\
\hline
\end{tabular}

list the corresponding results of the 32-zone runs, which include cases that we studied with high resolution, too.

\subsection{Dynamical evolution of reference model roo}

We begin with discussing the evolution of the disk in model r00, which will serve as reference model in this paper. Figure 2 shows the initial distributions of density, velocity, temperature, and electron fraction for this model. The obvious axisymmetric construction of these distributions was described above in the section on initial conditions. Figure 3 shows these distributions after $11.2 \mathrm{~ms}$. At this time the disk has reached a quasiequilibrium state, and its properties in model r00 are the following: within $100 \mathrm{~km}$ of the black hole the densities are a few times $10^{10} \mathrm{~g} \mathrm{~cm}^{-3}$, the temperatures are around $2 \mathrm{MeV}$, and the electron fraction is $0.04-0.06$.

Given our nested Cartesian grid, one cannot expect a precisely axisymmetric distribution to retain its symmetry when evolved with an explicit code. Some degree of numerical noise will always creep into the simulation and break the symmetry. This is expected to be on the scale of a couple of zones, so on a small scale as compared to the radius of the disk. On the other hand, the nested grid structure, specifically the boundaries of the grids and the transition to a lower resolution outside of a central cubic volume, will imprint a distinct 4-fold symmetry, which mostly remains small in amplitude. So, although it is known that disks also suffer physical instabilities, e.g. Papaloizou \& Pringle (1984), Kleiber \& Glatzel (1999), our simulations do not permit to connect the observed destruction of the initial axial symmetry with such physical instabilities. The numerical noise must be expected to drown all but the fastest growing high-order and low-order modes.

Figure 4 shows the temporal evolution of the black hole accretion rate and the integrated mass of the accretion torus. A phase of high mass accretion rate is visible from the outset until about $t \approx 10 \mathrm{~ms}$. This is a transient effect linked to the initial construction of the disk. This phase can also easily be spotted in the plot for the evolution of the maximum temperature in Fig. 6: the temperature increases transiently. The disk settles into a quasisteady state characterised by a nearly constant black hole accretion rate at a lower value and by only slowly changing values of the average density and temperature in the torus. This relaxation is more pronounced in the mass accretion rate of the black hole (Fig. 4), a fact which might indicate that it is the aftermath of the settling in the outer parts of the torus, which has now made its way to the centre.

\subsection{Influence of viscosity and varied disk mass}

The distributions of density, velocity, and temperature for models al3 and al4 are shown in Fig. 5. These have the same parameters as model r00 except for an increased viscosity (see Table 1). While both models show, in Fig. 6, a clear increase in disk temperature with increasing viscosity, the spatial distribution is different: model al 3 with the small value of the viscosity shows high temperatures between $4 \mathrm{MeV}$ and $8 \mathrm{MeV}$ only within a central region of radius $100 \mathrm{~km}$ (Fig. 5), whereas model al4 has $4 \mathrm{MeV}$ and higher in the whole extended disk. Since the pressure increases with temperature, this heating by the increased viscosity expands the disk and reduces its density (Fig. 5). Assuming 

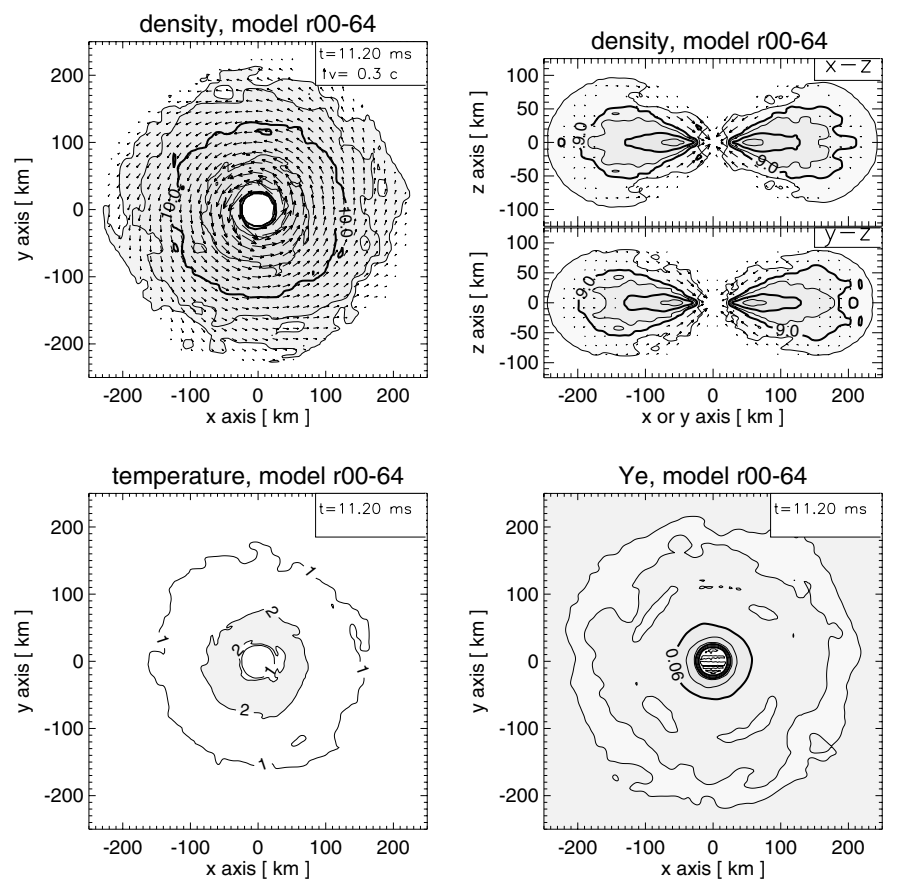

Fig. 3. Density distribution for models r00-64 in the orbital plane (left) and perpendicular to it (right) at about $11 \mathrm{~ms}$ after the start of the simulations. It is given in $\mathrm{g} \mathrm{cm}^{-3}$ with contours spaced logarithmically in steps of 0.5 dex. The arrows in the density plots indicate the velocity field. The temperature and electron fraction $\left(Y_{\mathrm{e}}\right)$ are displayed in the orbital plane. The temperature is measured in $\mathrm{MeV}$, its contours are labelled with the corresponding values. The contours of electron fraction are spaced linearly in steps of 0.02 .
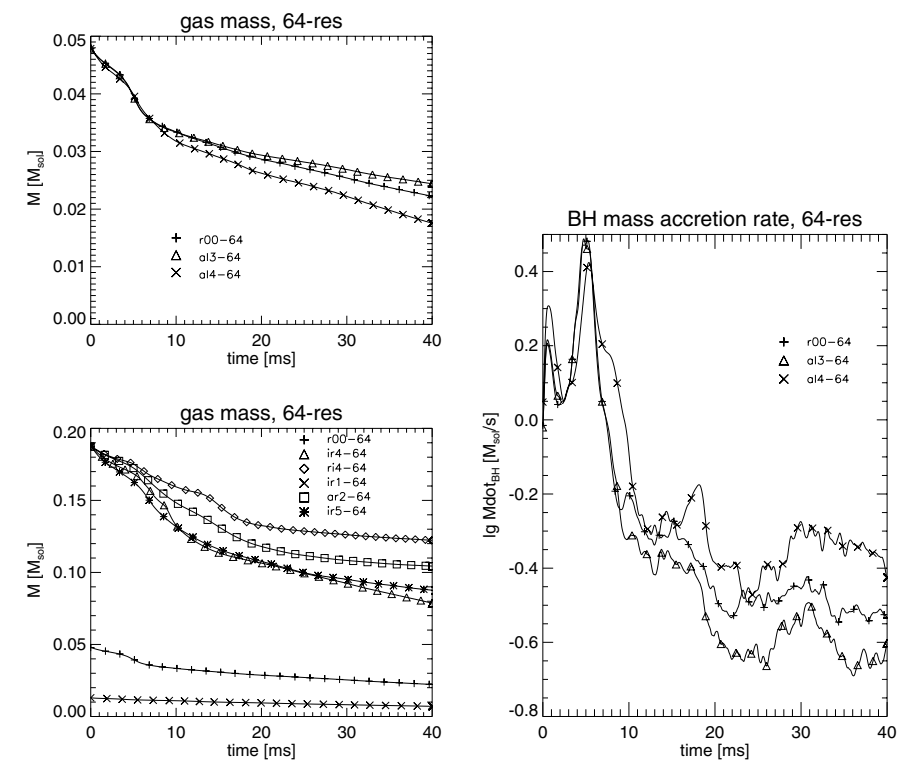

Fig. 4. Mass accretion rate of the black hole and and gas mass on the grid as functions of time for models r00-64, al3-64 and al4-64 with increasing disk viscosity. Gas mass on the grid as functions of time for the reference model r00-64, the low-mass torus model ir1-64, and the high-mass torus models ir4-64, ir5-64, ri4-64, and ar2-64.

otherwise equal velocities, a reduced density implies a reduced mass accretion rate. However, the large-scale effect of viscosity is to redistribute angular momentum from the centre of the disk out to the periphery, thus allowing mass to drift inward and be accreted by the black hole. The interplay of these two effects,
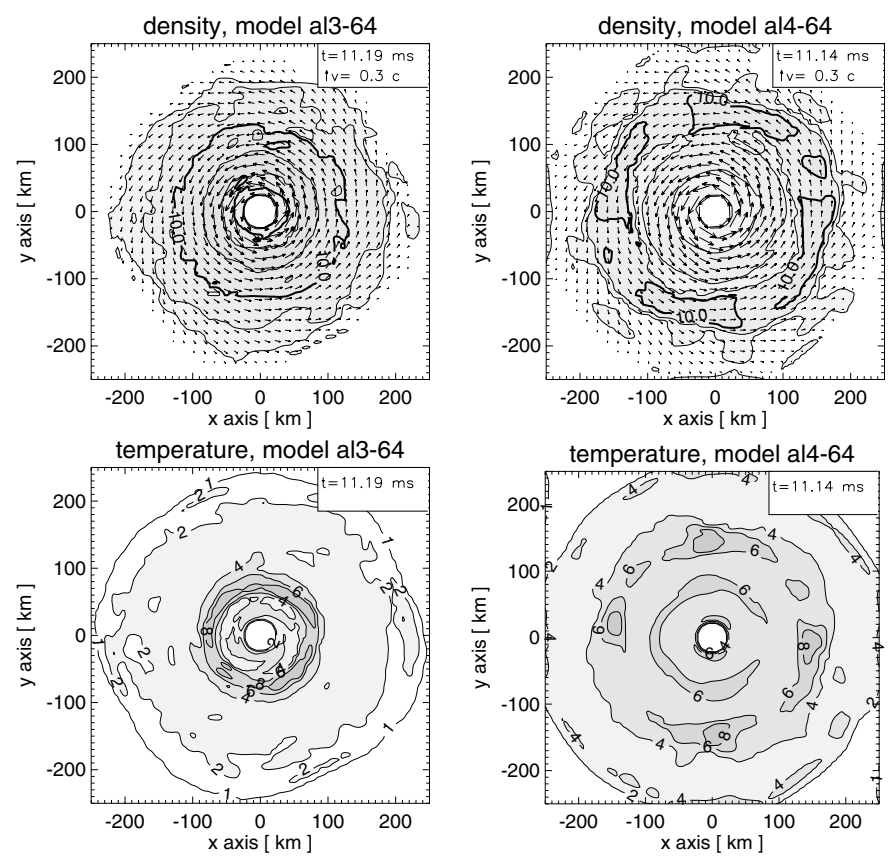

Fig. 5. Density and temperature distribution for models al3-64 (left) and al4-64 (right) with disk viscosities of $\alpha=0.01$ and $\alpha=0.1$, respectively. The density is given in $\mathrm{g} \mathrm{cm}^{-3}$ with contours spaced logarithmically in steps of 0.5 dex. The arrows in the density plots indicate the velocity field. The temperature is measured in $\mathrm{MeV}$, its contours are labelled with the corresponding values.
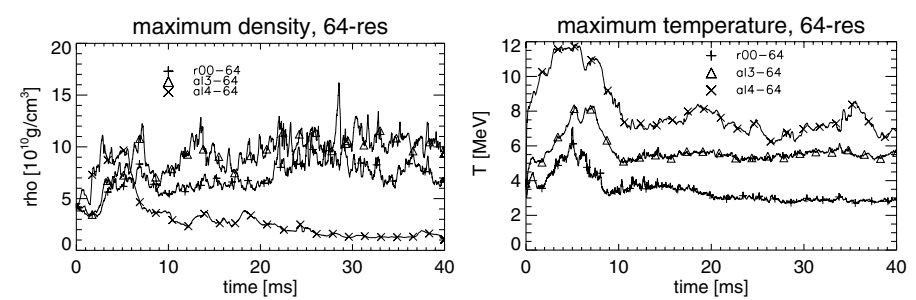

Fig. 6. Maximum values of gas density (left) and temperature (right) on the grid as functions of time for models r00-64, al3-64 and al4-64 with increasing disk viscosity.

the increase of the accretion due to viscosity on large scales and the decrease of the accretion rate due to a local decrease of the density in the innermost parts of the disk, can be observed in Fig. 4 for models al3 and al4. In model al3 the modest viscosity affects only the inner, most rapidly orbiting regions of the disk where shear effects are largest. The associated local central heating raises the thermal pressure and thus conspires to reduce the mass accretion rate. When the viscosity is further increased as done in model al4, the global viscosity effect dominates, and the mass accretion rate increases beyond the values of model r00 where no viscosity was added. As a consequence, the disk mass drops more rapidly than in the other models (Fig. 4), leading to a clearly lower maximum density in the disk (Fig. 6). The maximum disk temperature, on the other hand, is clearly largest in model al4 (Fig. 6).

The model pair ir4 and ir5, which also only differs in the amount of added viscosity, shows the same relationship with respect to the mass accretion rate as models r00 and al3: in Fig. 4 one can see a smaller mass accretion for the model with higher viscosity (ir5). The reasoning is similar to what has been described above: The temperatures are higher for model ir5, but 

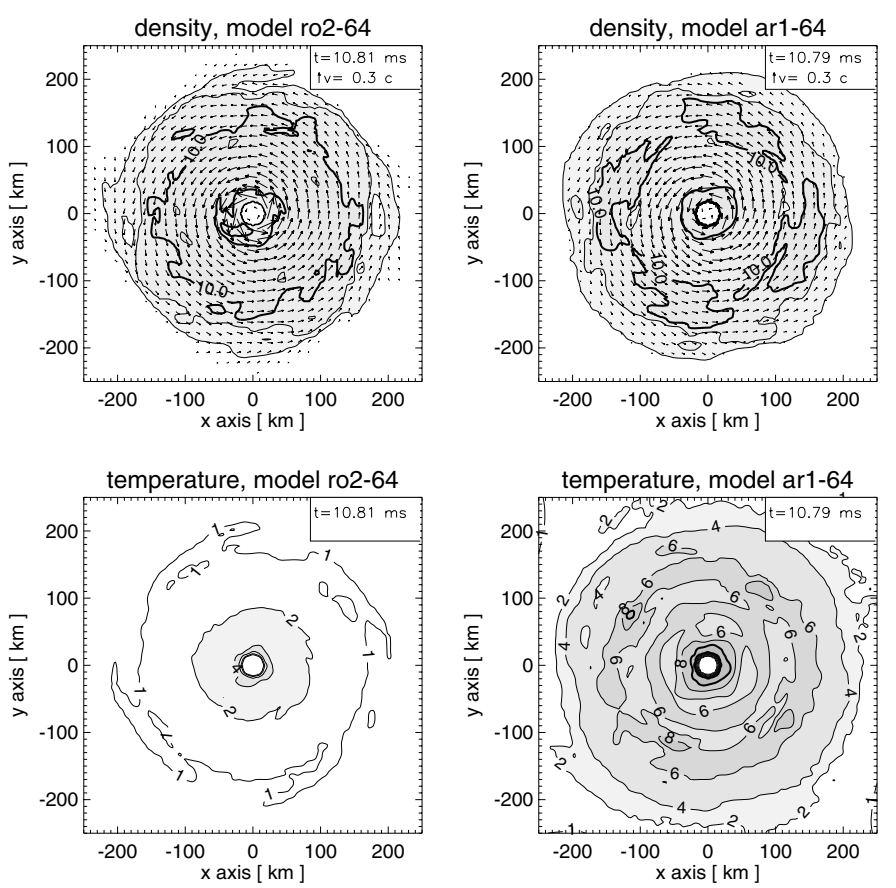

Fig. 7. Density and temperature distribution for models ro2-64 (left) and ar1-64 (right) for a corotating black hole, with disk viscosities of $\alpha=0.0$, and $\alpha=0.1$, respectively. The density is given in $\mathrm{g} \mathrm{cm}^{-3}$ with contours spaced logarithmically in steps of 0.5 dex. The arrows in the density plots indicate the velocity field. The temperature is measured in $\mathrm{MeV}$, its contours are labelled with the corresponding values.

the densities distinctly lower. It seems that for tori with higher mass, which is the case for models ir4 and ir5 relative to models $\mathrm{r} 00$, al3, and al4, the onset of the full effect of the increase of the mass accretion rate due to global angular momentum redistribution is offset to even higher values of the viscosity parameter. Increasing the disk mass by a factor of 4 does not produce any other major changes in flow dynamics. However the larger densities do have a large effect on the emission of neutrinos as will be described further below.

\subsection{Influence of black hole rotation and of a more compact gravitational potential}

We mimic some effects of relativistic gravity by using a Paczyński-Wiita (1980) potential and its extension to rotating black holes by Artemova et al. (1996). Varying the spin parameter between negative (counter-rotating black hole) and positive (black hole corotating with disk) values, the radius of the horizon changes and the ISCO is shifted to smaller values compared to the case of a black hole without rotation (Fig. 1). Looking at the central circle in Fig. 7 as compared to, e.g., model r00, one sees this slight shrinking of the inner grid boundary (at the arithmetic mean of $r_{\mathrm{H}}$ and $r_{\mathrm{ISCO}}$ ) when the black hole rotates in the prograde direction. Models ro2 and ro5 can be directly compared to r00, the only difference being the presence of rotation. The same applies to the pair al4 and ar1.

No major difference can be seen in the density or temperature distributions, except at the very centre close to the black hole. The temperatures for the rotating black holes are about $2 \mathrm{MeV}$ higher than in the non-rotating cases The counterrotating model ro5 retains the low temperatures of the reference model r00, i.e. around $2 \mathrm{MeV}$. In case of the viscous tori with corotating black hole the higher temperatures in the close
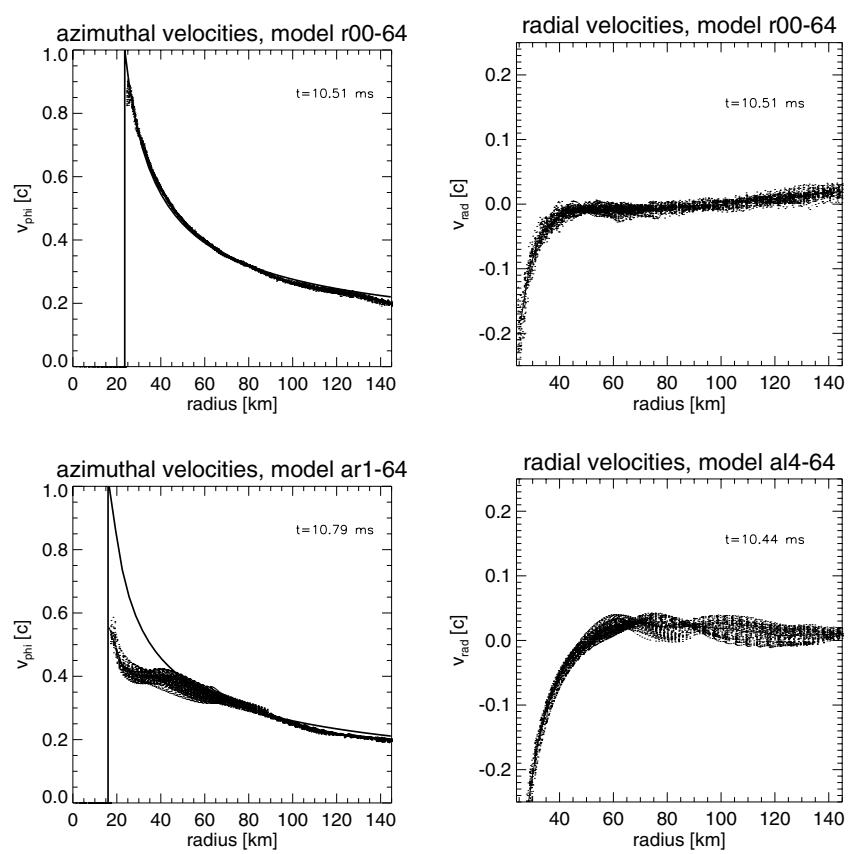

Fig. 8. Left panels: the dots represent the azimuthal velocities $v_{\varphi}(d)$ (normalised to the speed of light) of all zones in the equatorial plane of the reference model r00-64, and the high disk-viscosity model ar1-64 with corotating black hole, at the times after the start of the simulation indicated in the plots. The thin solid line gives the average value of the azimuthal velocities and the bold solid line the local Keplerian velocity $v_{\text {Kepler }}(d)$ of the Artemova et al. (1996) potential as a function of the equatorial radius $d$ (Eq. (3)). The inner vacuum boundary of the computational grid, is located at the arithmetic mean of the event horizon and innermost stable circular orbit (ISCO) of the central black hole. Right panels: the dots give the radial velocities $v_{r}(d)$ (normalised to the speed of light) as a function of the equatorial radius $d$ for all grid zones in the equatorial plane of the reference model r00-64 and the high diskviscosity model al4-64, at the same times. The solid lines represent the mean values of all zones within binning intervals of $3 \mathrm{~km}$.

vicinity of the black hole correlate with a distinct narrowing of the accretion torus in the vertical $(z)$ direction.

The combined increase of central temperatures brought about by viscosity and rotation has a clear impact on the velocities in the direct vicinity of the black hole. Figure 8 shows separately the azimuthal and radial velocities in the equatorial plane versus the distance from the centre of the black hole. Each zone is represented by a dot, and the spread of the points at a given radius reflects the deviations from rotational symmetry. For an axially symmetric configuration all dots at a specific radius would cluster on top of each other.

Comparing the radial velocities of models al4 and r00 (top and bottom right panels in Fig. 8), one sees that the large viscosity produces the inward facing accretion flow, i.e. negative velocities, at larger radii, inside of $60 \mathrm{~km}$ for model al 4 as opposed to $40 \mathrm{~km}$ for model $\mathrm{r} 00$. This reflects the canonical viscosity effect of globally shifting angular momentum outward allowing mass to fall inward. The added effect in model ar1 of narrowing the potential well with a rotating black hole does not change this situation significantly. Note that the outer parts of the tori show slow expansion at the displayed times, indicating ongoing relaxation as well as the consequence of outward transport of angular momentum.

The azimuthal velocities in model r00 closely follow the Keplerian values for the Paczyński-Wiita potential, see the top left panel in Fig. 8. Although the added viscosity in model al4 

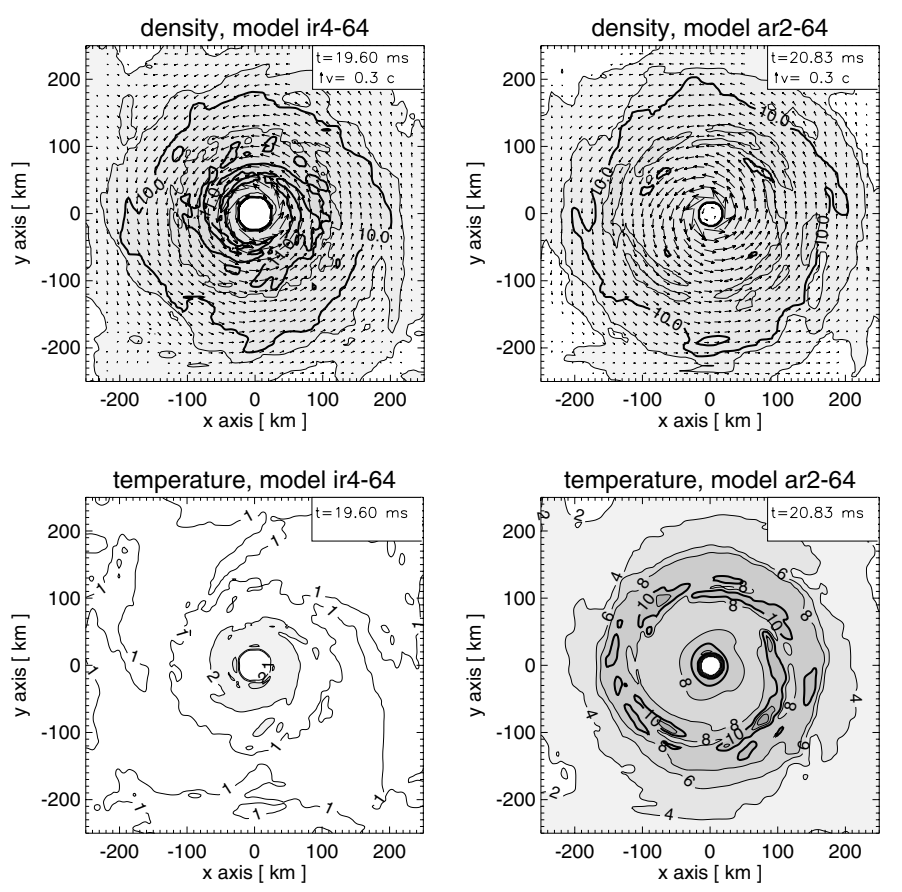

Fig. 9. Density and temperature distribution for high-mass torus models with nonrotating black hole and without disk viscosity (ir4-64; left) and with corotating black hole and disk viscosity $\alpha=0.1$ (ar2-64; right) at about $20 \mathrm{~ms}$ after the start of the simulations. The arrows indicate the velocity field. The density is given in $\mathrm{g} \mathrm{cm}^{-3}$ with contours spaced $\log$ arithmically in steps of 0.5 dex. The temperature is measured in $\mathrm{MeV}$, its contours are labelled with the corresponding values.

(bottom panel) increases the temperature in the disk, the pressure change due to this increase is not sufficient to appreciably change the velocity distribution. Only when black hole rotation is added, with its additional temperature increase and corresponding pressure increase in the vicinity of the black hole, does the matter in the disk become more strongly pressure supported within a radius of $50 \mathrm{~km}$. The azimuthal velocities are significantly below the Keplerian values in this region, as given by Eq. (3) (with the radius $r$ replaced by the equatorial distance $d$ ) for the ArtemovaBjörnsson-Novikov potential.

In all four pairs of models, r00/ro2, ri4/ir4, al4/ar1, and ir5/ar2 (see Figs. 9 for snapshots of the most massive torus models ir4 and ar2 at $t \approx 20 \mathrm{~ms}$ ) the first member of the pair is the non-rotating case, while the second contains a corotating black hole. All other parameters are kept equal. Examining Table 2 one notices that for each case the rotating model has a higher disk mass than the corresponding non-rotating model at the end of the simulated evolution (cf. also Fig. 4). This is a strong indication that a corotating black hole accretes matter from the initial tori less quickly, because the angular momentum at the ISCO is lower and a rotating black hole thus allows matter with lower angular momentum to remain on orbits. Moreover, the higher temperatures close to the black hole provide pressure support and additionally stabilise the torus. A confirmation is found by looking at the counter-rotating case ro5, which can be compared to models r00 and ro2. Indeed, the disk of model ro5 has an even smaller mass than that of model r00.

\subsection{Neutrino emission}

The neutrino luminosities and cumulative energy emitted in neutrinos as functions of time for a sample of 64-resolution
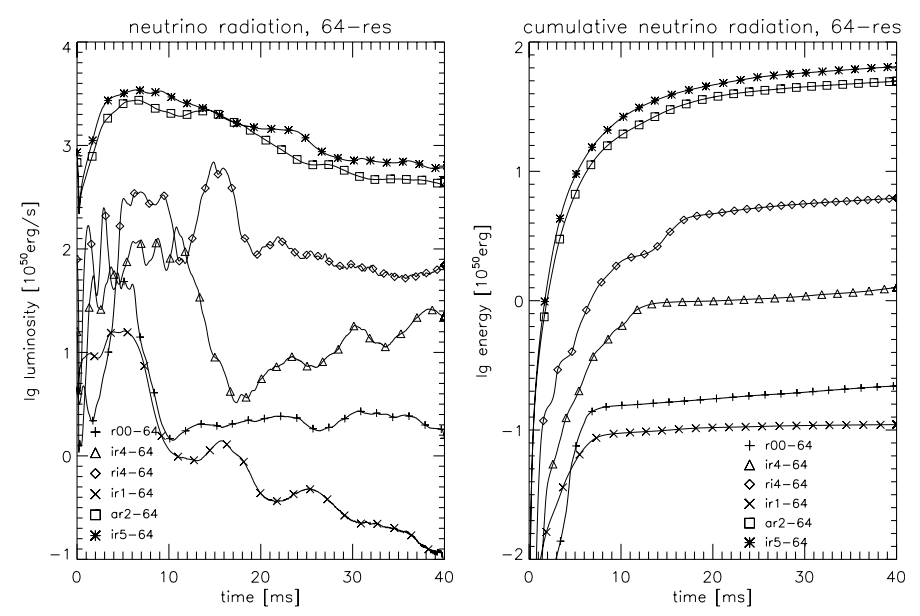

Fig. 10. Total neutrino luminosities (left) and cumulative energy radiated in neutrinos (right) as functions of time for the reference model r00-64, the low-mass torus model ir1-64, and the high-mass torus models ir4-64, ir5-64, ri4-64, and ar2-64.

models are shown in Fig. 10. Note that electron neutrinos, $v_{\mathrm{e}}$, and electron antineutrinos, $\bar{v}_{\mathrm{e}}$, dominate the emission by a large factor, because at the density and temperature conditions in the accretion tori their production rate by charged-current absorption of electrons and positrons on free protons and neutrons, respectively,

$$
\begin{aligned}
& \mathrm{e}^{-}+\mathrm{p} \longrightarrow \mathrm{n}+v_{\mathrm{e}} \\
& \mathrm{e}^{+}+\mathrm{n} \longrightarrow \mathrm{p}+\bar{v}_{\mathrm{e}},
\end{aligned}
$$

is much higher than the production rate of neutrino-antineutrino pairs of all flavors by thermal neutrino processes and nucleonnucleon bremsstrahlung (see also Ruffert \& Janka 1999; Rosswog \& Liebendörfer 2003).

The $\bar{v}_{\mathrm{e}}$ luminosities are initially much larger than the $v_{\mathrm{e}}$ luminosities and at the end of the calculated evolution still typically 2-3 times higher (see Table 2). The tori consist of decompressed neutron star matter, which inside of the neutron star was initially in a very neutron-rich $\left(Y_{\mathrm{e}} \lesssim 0.1\right)$ state of neutrinoless beta equilibrium (the initial setup chosen for our simulations was adapted to neutron star merger results and therefore still reflects this origin of the torus matter, cf. Fig. 2). Chemical or kinetic equilibrium at densities much below nuclear matter corresponds to larger values of $Y_{\mathrm{e}}$ and a higher proton abundance. At decompression, matter tries to approach this new equilibrium state, in which the production of $v_{\mathrm{e}}$ and $\bar{v}_{\mathrm{e}}$ are in balance. During this "protonization" electron antineutrinos are emitted in larger numbers than $v_{\mathrm{e}}$. The consequences of this process are visible in two ways. First, comparing the $Y_{\mathrm{e}}$ distribution in Fig. 11 after $11 \mathrm{~ms}$ and $20 \mathrm{~ms}$ of evolution with the initial state shown in Fig. 2 one sees significantly higher values of $Y_{\mathrm{e}}$, i.e. more protons. Secondly, in Fig. 12, the comparison of the local energy emission rates per unit area or volume, of $v_{\mathrm{e}}, \bar{v}_{\mathrm{e}}$, and heavylepton neutrinos plus antineutrinos (" $v_{x}$ " for the sum of muon and tau neutrinos, $v_{\mu}, \bar{v}_{\mu}, v_{\tau}$, and $\bar{v}_{\tau}$ ), respectively, shows that the $\bar{v}_{\mathrm{e}}$ emission is significantly higher than that of $v_{\mathrm{e}}$. The protonization is faster and leads to higher values of $Y_{\mathrm{e}}$ at the end of our simulations in case of hotter and less dense tori; this trend is very similar for larger viscosity and black hole rotation (models ro2, ar1, ir4 and ar2 in Fig. 11). All tori are at most marginally opaque to neutrinos in their denser parts and the diffusion times short in most regions. The $Y_{\mathrm{e}}$ evolution is therefore governed by 

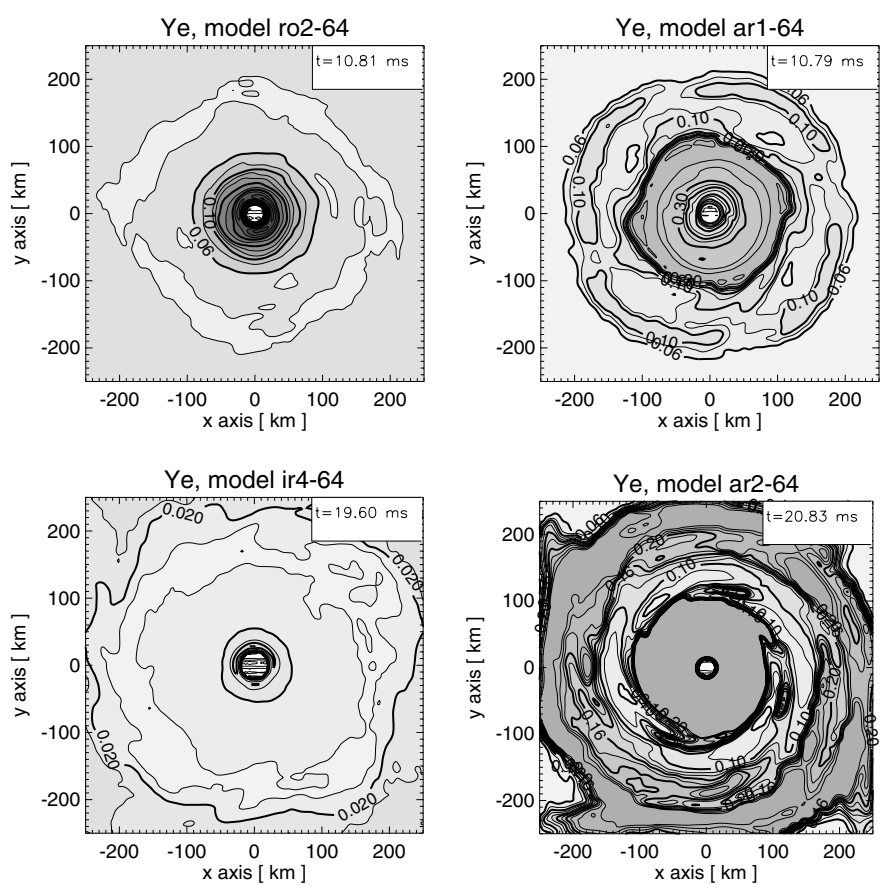

Fig. 11. Electron fraction in the orbital plane for models ro2-64, ar1-64, ir4-64 and ar2-64, in the orbital plane at about $11 \mathrm{~ms}$ after the start of the simulations. The contours are spaced linearly in steps of 0.02 .

the capture rates directly. Since positron captures in reaction (9) increase extremely steeply with rising temperature, the highest values of $Y_{\mathrm{e}}$ develop in regions of highest temperatures.

The mean energies of the radiated neutrinos $\left(\left\langle\epsilon_{v_{\mathrm{e}}}\right\rangle,\left\langle\epsilon_{\bar{v}_{\mathrm{e}}}\right\rangle\right.$, and $\left.\left\langle\epsilon_{v_{x}}\right\rangle\right)$ are defined as the ratio of integral energy loss (rate) to total number loss (rate) in neutrinos of a given kind (see Ruffert et al. 1996, 1997). They correlate with the average and maximum torus temperatures as visible from the parallel trends of $T_{\max }$ and the mean neutrino energies in Table 2. Electron neutrinos and antineutrinos are radiated away with mean energies of $10-20 \mathrm{MeV}$ and are similarly energetic, because electron degeneracy plays a minor role and the cross sections of both production reactions scale equally with particle energies; moreover, reabsorption of neutrinos is of minor importance because the tori are essentially transparent to neutrinos. Heavy-lepton neutrinos, on the other hand, tend to have a slightly lower mean energy except in cases with the largest torus masses (models ri4, ir4, ir5, and ar2), which also tend to be among the hottest models. Only in the two cases with the highest torus temperatures and shear viscosity parameter $\alpha=0.1$ (models ir5 and ar2) do muon and tau neutrinos yield a non-negligible contribution to the total energy loss in neutrinos.

The results summarised in Tables 2 and 3 and Fig. 10 show that the neutrino luminosities rise steeply with torus mass. In simulations without shear viscosity $(\alpha=0)$ we find a roughly quadratic increase, $L_{v} \propto M^{2}$ (compare model r00-64 with ir4-64 and ro2-64 with ri4-64), whereas with shear viscosity $\alpha=0.1$ a certain saturation can be observed and the luminosities increase only slightly steeper than linearly, $L_{v} \propto M^{1+\xi}$ for $\xi>0$ (models al4-64 vs. ir5-64 and ar1-64 vs. ar2-64). Shear viscosity has the most dramatic influence on the neutrino emission: the luminosities grow by a factor of 30-40 when $\alpha=0.1$ instead of $\alpha=0$ (models r00-64 vs. al4-64; ir4-64 vs. ir5-64). In contrast, a black hole with a spin parameter up to $a \sim 0.6$ in corotation with the torus has a much weaker influence on the neutrino emission. While for calculations without shear viscosity $(\alpha=0)$ the
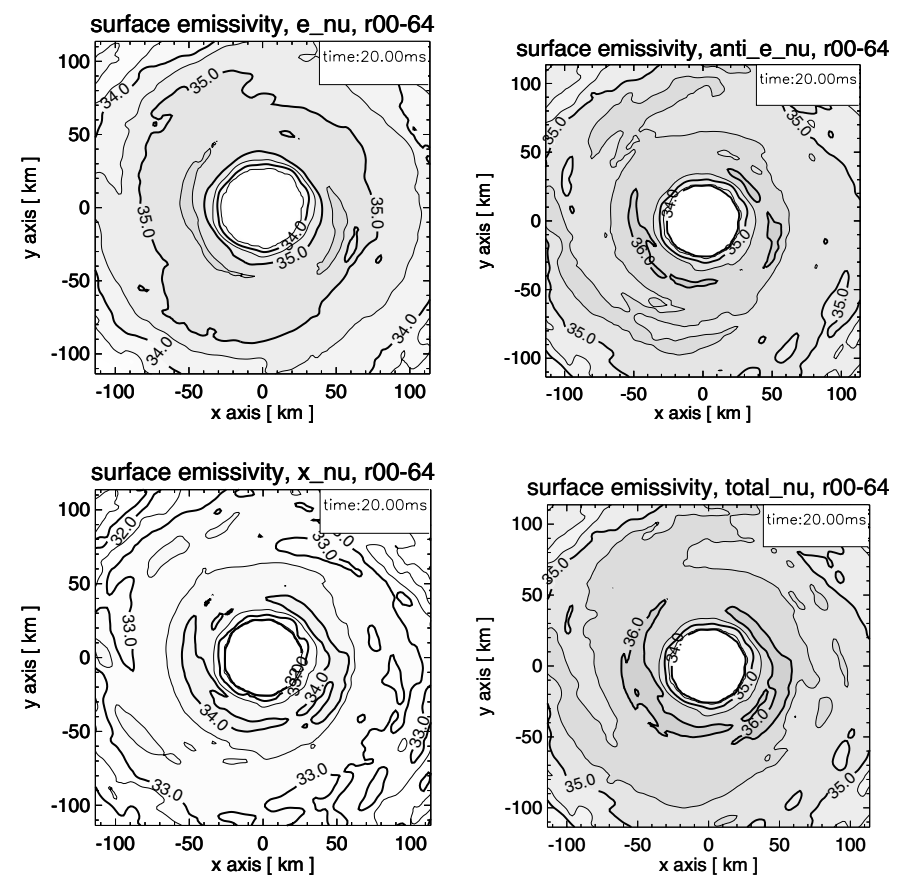

Fig. 12. Neutrino energy loss rates per unit area in the orbital plane for model r00-64 at $20 \mathrm{~ms}$ after the start of the simulations. The plotted values show the logarithm of the rates in $\mathrm{erg} \mathrm{cm}^{-2} \mathrm{~s}^{-1}$, obtained by integration of the local energy loss rates per unit of volume from $z=0$ to infinity. The top left panel gives the results for electron neutrinos, the top right panel for electron antineutrinos, the lower left panel for the sum of muon and tau neutrinos and antineutrinos, and the lower right panel the total values for neutrinos and antineutrinos of all flavors. The contours are spaced in steps of $0.5 \mathrm{dex}$, bold lines are labelled with their corresponding values. The grey shading emphasises the emission levels, dark grey representing the largest energy loss by neutrino emission.

Table 3. Torus mass $M_{\mathrm{d}}$, black hole mass accretion rate $\dot{M}_{\mathrm{d}}$, estimated accretion time scale of the torus, $t_{\mathrm{acc}} \equiv M_{\mathrm{d}} / \dot{M}_{\mathrm{d}}$, total neutrino luminosity $L_{v}$, integral rate of energy deposition by neutrino-antineutrino annihilation around the accretion torus, $\dot{E}_{v \bar{v}}$, and total energy deposition by $v \bar{v}$ annihilation in the time interval $t_{\text {fin }}, E_{\nu \bar{v}}$, for all 64-resolution models. All quantities are given at time $t_{\text {fin }}$ after the start of the simulation.

\begin{tabular}{cccccccc}
\hline \hline Model & $\begin{array}{c}t_{\text {fin }} \\
\mathrm{ms}\end{array}$ & $\begin{array}{c}M_{\mathrm{d}} \\
10^{-2} M_{\odot}\end{array}$ & $\begin{array}{c}\dot{M}_{\mathrm{d}} \\
M_{\odot} \mathrm{s}^{-1}\end{array}$ & $\begin{array}{c}t_{\mathrm{acc}} \\
\mathrm{ms}\end{array}$ & $\begin{array}{c}L_{v} \\
10^{50} \frac{\mathrm{erg}}{\mathrm{s}}\end{array}$ & $\begin{array}{c}\dot{E}_{v \bar{v}} \\
10^{50 \frac{\mathrm{erg}}{\mathrm{s}}}\end{array}$ & $\begin{array}{c}E_{v \bar{v}} \\
10^{50} \mathrm{erg}\end{array}$ \\
\hline r00-64 & 40.0 & 2.22 & 0.29 & 77. & 1.8 & $1.8 \times 10^{-4}$ & $4.4 \times 10^{-4}$ \\
al3-64 & 40.0 & 2.44 & 0.25 & 98. & 11.4 & $8 \times 10^{-3}$ & $3.8 \times 10^{-3}$ \\
a14-64 & 40.0 & 1.76 & 0.38 & 46. & 80.0 & 0.4 & 0.26 \\
ri4-64 & 40.0 & 12.22 & 0.41 & 298. & 70.0 & 0.35 & 0.13 \\
ro2-64 & 40.0 & 3.10 & 0.16 & 194. & 3.4 & $7.2 \times 10^{-4}$ & $3.3 \times 10^{-3}$ \\
ro5-64 & 40.0 & 1.13 & 0.25 & 45. & 1.0 & $3 \times 10^{-5}$ & $1.8 \times 10^{-4}$ \\
ir1-64 & 40.0 & 0.71 & 0.11 & 65. & 0.1 & $5 \times 10^{-7}$ & $8 \times 10^{-5}$ \\
ir4-64 & 40.0 & 7.87 & 1.28 & 61. & 22.4 & $2.8 \times 10^{-2}$ & $1.8 \times 10^{-3}$ \\
ir5-64 & 40.0 & 8.77 & 0.58 & 151. & 610.0 & 21.0 & 2.40 \\
ar1-64 & 40.0 & 2.34 & 0.35 & 67. & 110.0 & 0.7 & 0.29 \\
ar2-64 & 40.0 & 10.44 & 0.28 & 373. & 440.0 & 11.0 & 1.90 \\
\hline
\end{tabular}

neutrino luminosities increase at most by a factor of 2-3 (compare model r00-64 with ro2-64 and model ri4-64 with ir4-64 in Table 2 and Fig. 10), an additional increase of the neutrino emission is hardly visible or absent for cases with $\alpha=0.1$ (compare models al4-64 with ar1-64 and models ir5-64 with ar2-64). Black hole rotation with more extreme spin parameter, however, leads to a large boost of the neutrino emission as can be seen from models ro2-32 with $a=0.6$ and ro3-32 with $a=0.8$ in 
Table 2. Despite of nearly equal torus masses, the latter model has a 9 times higher neutrino luminosity.

The series of models with reduced resolution exhibits the tendency of slightly lower torus temperatures compared to the corresponding simulations with finer grid zoning at the same time of the evolution. The neutrino luminosities and mean energies, however, show no clear trend and can be higher or lower, depending mostly on whether the torus has a larger or smaller mass than in the better resolved calculations. This sensitivity together with the fact that the less massive tori have lost most of their mass to the black hole after an evolution of $70 \mathrm{~ms}$ (Table 2), disfavours the late-time models with lower resolution for a quantitative comparison of the influence of viscosity and black hole rotation. The neutrino emission during these late stages has dropped to a low level in most cases and is therefore mostly dependent on the torus mass which is left around the black hole (see Table 2). The general trends associated with viscosity, torus mass, and black hole rotation, however, can also be seen in the 32-resolution models.

The upper left panels of Fig. 13 and right panel of Fig. 14 provide information about the instantaneous conversion efficiency of rest-mass energy to neutrino-antineutrino energy,

$q_{v} \equiv \frac{L_{v}}{\dot{M} c^{2}}$,

for a selection of 64-resolution models as function of time. It is obvious that the values are extremely low $\left(q_{v} \ll 1 \%\right)$ in case of zero physical shear viscosity $(\alpha=0)$ and become of order $1 \%$ only for large torus masses $\left(M_{\mathrm{d}}>0.1 M_{\odot}\right.$ as in case of model ri4-64, cf. Table 3). For $\alpha=0.1$ the efficiencies reach several percent (e.g. model ar1) and models ar2 and ir5 in Fig. 13). It is interesting that $q_{v}$ for the low-mass torus model ar1 reaches its maximum at early times and then decreases towards the end of our simulations, whereas the values are stable or even increase over the simulated period of evolution in models ir5 and ar2.

\subsection{Neutrino-antineutrino annihilation}

Energy deposition in the vicinity of the black-hole torus system by the annihilation of neutrinos $(v)$ and antineutrinos $(\bar{v})$ of all flavors, which are radiated away from the accretion torus, to electron-positron pairs in the process

$v+\bar{v} \longrightarrow \mathrm{e}^{+} \mathrm{e}^{-}$

is considered as a way to produce a highly relativistic pairplasma, if the density of baryonic matter in the deposition region is sufficiently low. Provided the entrainment of additional baryons during the subsequent expansion can be prevented, the pressure by radiation and electron-positron pairs can then accelerate the plasma to ultrarelativistic velocities. Recent general relativistic hydrodynamic simulations have demonstrated that this indeed happens and that the ultrarelativistic outflow becomes collimated to semi-opening angles between $\sim 5^{\circ}$ and $\sim 12^{\circ}$ (Aloy et al. 2005).

Because we calculate energy and lepton number loss in our 3D hydrodynamic simulations of the torus evolution by a neutrino trapping scheme, but do not solve the neutrino-transport problem, the corresponding energy deposition is evaluated for our hydrodynamic models in a post-processing step. The numerical procedure for doing this is rather CPU-time consuming and explained in detail in Ruffert \& Janka $(1999,2001)$ and Ruffert et al. (1997). The energy deposition rate per unit volume, $\dot{Q}_{v \bar{v}}$, at a point $\boldsymbol{r}$ is calculated as the sum of the contributions from
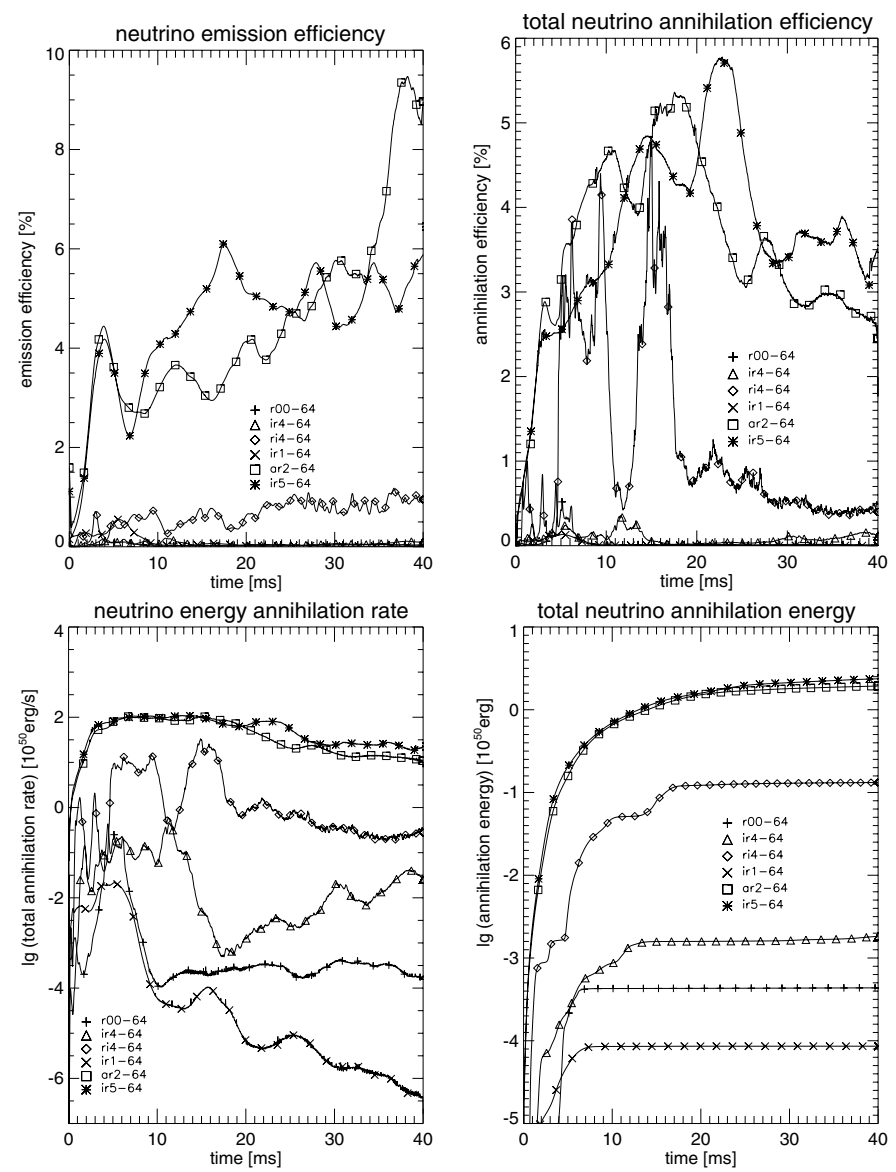

Fig. 13. Conversion efficiency of rest-mass energy to neutrinos, $q_{v} \equiv$ $L_{v} /\left(\dot{M} c^{2}\right)$ (top left), conversion efficiency of neutrino energy to electronpositron pairs by neutrino-antineutrino annihilation, $q_{v \bar{v}} \equiv \dot{E}_{v \bar{v}} / L_{v}$ (top right), integral rate of energy deposition by neutrino-antineutrino annihilation around the accretion torus, $\dot{E}_{\bar{v}}$ (bottom left), and cumulative energy deposition by $v \bar{v}$ annihilation, $E_{\gamma \bar{v}}$ (bottom right), as functions of time for the same set of models shown in Figs. 4, and 10.

neutrinos and antineutrinos emitted from all regions of the accretion torus, thus taking into account the specific emission geometry and local variations of the neutrino loss rates as visible in the emission maps of Figs. 12. Neutrinos and antineutrinos radiated from the hot accretion torus interact with each other in the surroundings with a finite probability, which depends on the number densities and the energies of these neutrinos and on the angle between the directions of neutrino and antineutrino propagation (see, e.g., Goodman et al. 1987; Ruffert et al. 1997). The total energy deposition rate,

$\dot{E}_{\bar{\nu}}=C L_{v} L_{\bar{v}}\left(\frac{\left\langle\epsilon_{\nu}^{2}\right\rangle\left\langle\epsilon_{\bar{v}}\right\rangle+\left\langle\epsilon_{\bar{v}}^{2}\right\rangle\left\langle\epsilon_{v}\right\rangle}{\left\langle\epsilon_{v}\right\rangle\left\langle\epsilon_{\bar{v}}\right\rangle}\right)$,

therefore increases with the product of neutrino and antineutrino luminosities and with the spectrally averaged neutrino energies, times a factor $C$ that accounts for the dependence on the angular distribution of the neutrinos. The quantities $\left\langle\epsilon_{v}\right\rangle$ and $\left\langle\epsilon_{v}^{2}\right\rangle$ in Eq. (12) denote the mean energies and mean squared energies of $v$ and $\bar{v}$. The factor $C$ contains the weak interaction coefficients and terms that depend on the geometry of the neutrino-emitting torus region. This factor is a function of the emission geometry and drops steeply with the distance from the neutrino source.

Large collision angles between neutrinos and antineutrinos are found only close to the source and therefore annihilation is 

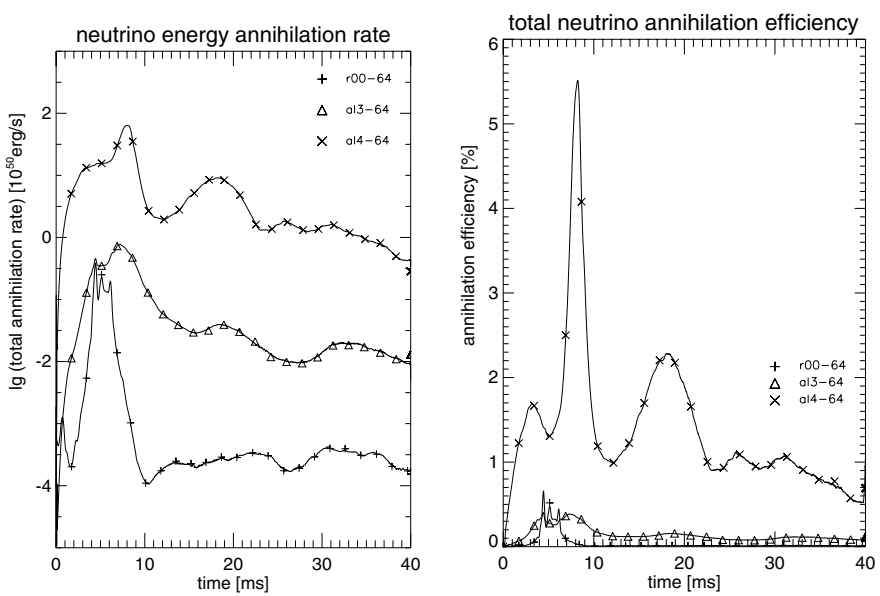

Fig. 14. Integral rate of energy deposition by neutrino-antineutrino annihilation around the accretion torus, $\dot{E}_{v \bar{v}}(l e f t)$ and conversion efficiency of neutrino energy to electron-positron pairs by neutrino-antineutrino annihilation $q_{v \bar{v}} \equiv \dot{E}_{v \bar{v}} / L_{v}$ (right), as functions of time for selected 64-resolution models with the same torus mass but different torus viscosity. The models are the same as in Figs. 4 and 6.

favoured near the hottest parts of the torus, close to the equatorial plane. There, however, the gas densities are still high and the energy deposition by $v \bar{v}$ annihilation is compensated by very rapid cooling due to the charged-current reactions of Eqs. (8) and $(9)^{1}$. Neutrino-antineutrino annihilation is therefore not efficient in establishing net energy transfer to regions with large baryon densities. On the other hand, the annihilation rate is also higher above the poles of the black hole, where neutrinos emitted from the torus have a high probability to collide with large angles in low-density environment. This can be seen in the four panels of Fig. 15, which show the annihilation rate in the $d$-z-planes perpendicular to the orbital plane for models r00-64, ro2-64, ro5-64, and ar2-64 at $20 \mathrm{~ms}$ after the start of the simulations.

The corresponding integral rate of energy deposition by neutrino-antineutrino annihilation around the accretion torus, $\dot{E}_{v \bar{v}}$, the cumulative energy deposition by $v \bar{v}$ annihilation, $E_{v \bar{v}}$, and the neutrino conversion efficiency,

$q_{\nu \bar{v}} \equiv \dot{E}_{v \bar{v}} / L_{v}$

as functions of time are plotted for some of our computed models in Figs. 13 and 14. Values of $\dot{E}_{\nu \bar{v}}$ and $E_{\nu \bar{\gamma}}$ for all 64-resolution models at the end of the simulations are listed in Table 3 .

The contributions from muon and tau neutrinos and antineutrinos can safely be ignored in this evaluation, because their luminosities are much lower (see Table 2) and their annihilation cross sections are also smaller by a factor of about 5 compared to those of electron neutrinos and antineutrinos. The geometry of the neutrino-emitting torus region and therefore the factor $C$ does not change strongly during the quasi-stationary phase of the torus evolution and therefore $C$ can be calibrated by evaluating the $v \bar{v}$ annihilation in detail at one or more representative times. Thus Eq. (12) with constant $C$ allows for a good approximation of the time evolution of $\dot{E}_{\gamma \bar{v}}$ (as shown in Figs. 13 and 14) by using the neutrino luminosities and mean neutrino energies as determined from the torus simulations, without the need to perform the CPU-time demanding post-processing for the $v \bar{v}$ annihilation maps of Fig. 15 many times for our sample of runs.

1 The energy loss associated with the reemission of neutrinos by charged-current reactions is not taken into account in Fig. 15.
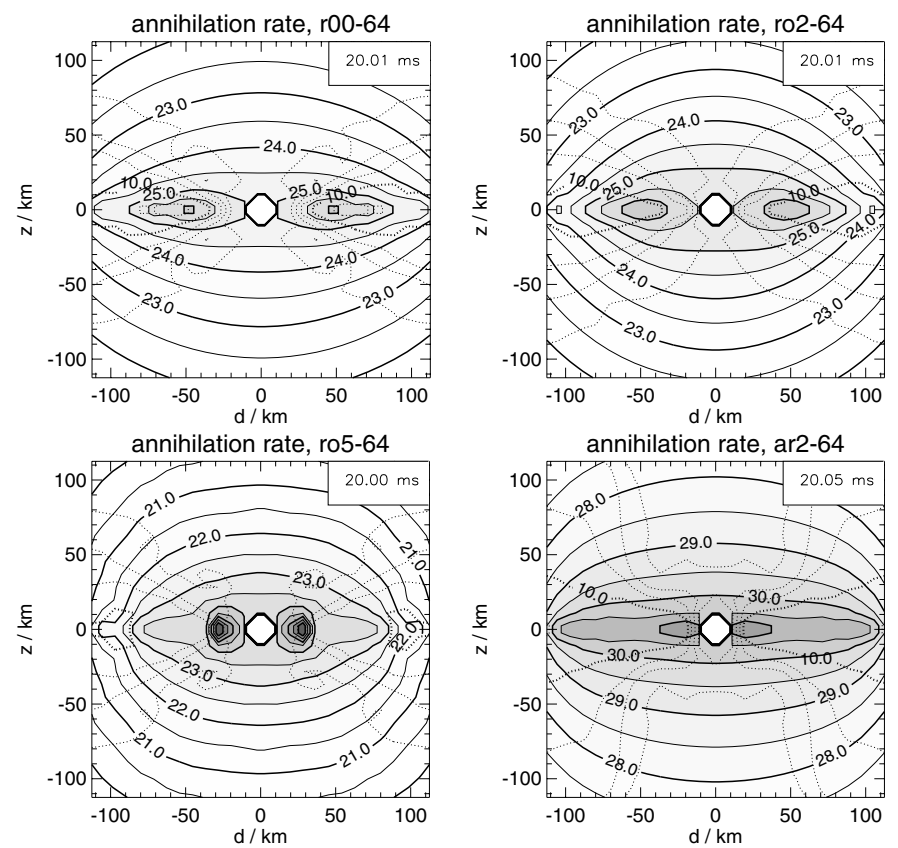

Fig. 15. Maps of the local energy deposition rates (in $\operatorname{erg~cm}^{-3} \mathrm{~s}^{-1}$ ) by $v \bar{v}$ annihilation to $\mathrm{e}^{+} \mathrm{e}^{-}$pairs in the surroundings of the accretion torus for models r00-64, ro2-64, ro5-64, and ar2-64 at a time $20 \mathrm{~ms}$ after the start of the simulation. The solid contour lines in a plane perpendicular to the equatorial plane represent values averaged over azimuthal angles around the z-axis. The contours are logarithmically spaced in steps of 0.5 dex and the grey shading emphasises the levels with dark grey meaning high energy deposition rate. The energy deposition rate was evaluated only in that region around the torus where the baryon mass density is below $10^{11} \mathrm{~g} \mathrm{~cm}^{-3}$. The white octagonal area around the centre with a semidiameter of one Schwarzschild radius indicates the presence of the central black hole. The dotted contour lines represent levels of constant values of the azimuthally averaged mass density, also logarithmically spaced with intervals of $0.5 \mathrm{dex}$; the bold dotted line corresponds to $\rho=10^{10} \mathrm{~g} \mathrm{~cm}^{-3}$.

Only our most massive tori possess initially neutrino-opaque "cores" with densities above $\sim 10^{11} \mathrm{~g} \mathrm{~cm}^{-1}$, whereas in the other cases such regions are absent. When the optical depths for neutrinos are larger than unity and neutrinospheres exist, these typically have ellipsoidal or egg-shaped cross sections in the $d-z$. plane. Also in our massive tori the opaque cores disappear and the neutrino optical depths fall below unity everywhere after the tori have lost some mass to the black hole and/or thermal inflation by viscous heating has decreased the density. All of the tori shown in Fig. 15 are therefore transparent to neutrinos. A comparison of the annihilation maps of models $\mathrm{r} 00$, ro2, and ro5 in Fig. 15 shows the influence of the black hole rotation. The values of the energy deposition rates differ and scale roughly with the neutrino luminosity as expected from Eq. (12) (cf. also Table 3), but the morphology of the contour lines which envelope the black-hole torus system is very similar. In the counterrotating case (model ro5) the two central maxima are more pronounced and represent a toroidal volume which is more compact than in the other cases.

Only our most massive tori (models ri4, ir4, ir5, and ar2) and the viscous intermediate-mass models al 4 and ar1 reveal $v \bar{v}$ annihilation rates $\dot{E}_{\nu \bar{v}}$ which become larger than $10^{50} \mathrm{erg} \mathrm{s}^{-1}$ at least for some period of the simulated evolution (see Figs. 13 and 14; the non-viscous massive torus model ir4, however, only marginally reaches this value). The numbers listed in Table 3 clearly show the correlation with the neutrino luminosities. They 
also show that the black hole rotation (up to a rotation parameter of $a=0.6$ ) has hardly any influence on the total energy $E_{v \bar{v}}$ deposited by $v \bar{v}$ annihilation (compare the model pairs r00 and ro2, al4 and ar1, and ir5 and ar2). In contrast, the torus mass makes a big effect for viscous (model pairs al4 and ir5, ar1 and ar2) as well as nonviscous cases (model pairs r00 and ir4, ro2 and ri4); in case of the higher torus masses $E_{\nu \bar{v}}$ is larger by factors of 5-40. Again shear viscosity ( $\alpha=0.1$ instead of $\alpha=0$ ) makes the biggest difference. For the latter, viscous models of the pairs (ri4,ar2), (ir4,ir5), and (r00,al4) $E_{\nu \bar{v}}$ is higher by factors between 15 and 1000. The lower right panel of Fig. 15 shows the annihilation map for one of the two most extreme cases, model ar2, at $20 \mathrm{~ms}$ after the start of the simulation when the integral rate of energy conversion by $v \bar{v}$ annihilation was $\dot{E}_{\nu \bar{v}}=5.8 \times 10^{51} \mathrm{erg} \mathrm{s}^{-1}$. The energy deposition rates above the poles of the black hole reach $10^{30} \mathrm{erg} \mathrm{cm}^{-3} \mathrm{~s}^{-1}$ at this time. Even at $z$-distances of $50 \mathrm{~km}$ they are still more than $10^{29} \mathrm{erg} \mathrm{cm}^{-3} \mathrm{~s}^{-1}$. For the most massive of our tori and the viscous tori with intermediate masses, the annihilation efficiencies $q_{v \bar{v}}$ can reach several percent, peaking for short times at more than 5\% (Figs. 13 and 14). We note that the results for $v \bar{v}$ annihilation which we have obtained for the 32-resolution runs are in general smaller by about $20 \%$ compared to the better resolved models. We also point out that the total energy deposition by $v \bar{v}$ annihilation $\left(E_{v \bar{v}}\right)$ of model ir5-64 at the end of our simulations (at $40 \mathrm{~ms}$ ) is about $25 \%$ higher than that of model ar2-64, but we estimate the remaining torus lifetime of model ar2-64 to be more than two times longer.

At the end of the simulated evolution, the tori have lost typically about half of their mass to the black hole, and the mass accretion rates have decreased to $0.1-0.4 M_{\odot} \mathrm{s}^{-1}$ in most cases. The total neutrino luminosities have declined from their peak values by roughly an order of magnitude for the tori with intermediate mass and by about a factor of 5 for our most massive cases (Fig. 10 and Table 3). Correspondingly, the $v \bar{v}$ annihilation rates have dropped by roughly two orders of magnitude below their maximum values in the former case (Fig. 14), while in the latter case they have decreased only by a factor of 5-10 (models ar2 and ir5 in Fig. 13, which have peak values of $\dot{E}_{\bar{\gamma}} \gtrsim 10^{52} \mathrm{erg} \mathrm{s}^{-1}$ ). This is less steep than the square of the total neutrino luminosities and suggests a time-dependent change of the emission of electron neutrinos relative to electron antineutrinos (note the dependence of $\dot{E}_{\nu \bar{v}}$ on the product $L_{\nu} L_{\bar{v}}$ in Eq. (12)). This fact is consistent with the large rise of $Y_{\mathrm{e}}$ relative to the initially low values (e.g. visible in the comparison of Fig. 11 with Fig. 2). When $Y_{\mathrm{e}}$ climbs closer to 0.5 and thus tries to approach a value where kinetic equilibrium of $v_{\mathrm{e}}$ and $\bar{v}_{\mathrm{e}}$ production holds, the initially much stronger emission of $\bar{v}_{\mathrm{e}}$ evolves to a more balanced release of electron neutrinos and antineutrinos ${ }^{2}$.

\section{Axial low-density funnel and GRB production}

Near the poles of the black hole and along the system axis, the density in our simulations decreases quickly. Material which is swept into these polar regions during and immediately after the merging of the double neutron stars or neutron-star black-hole binary falls into the (newly formed) black hole within a freefall time scale, because it is not supported by a strong negative pressure gradient or centrifugal forces. Thus a cylindrical funnel along the rotation axis of the black-hole torus system is rapidly "evacuated". The continuous entrainment of more gas from the

\footnotetext{
${ }^{2}$ Note that the product $L_{v} L_{\bar{\nu}}$ has a maximum for $L_{v}=L_{\bar{\nu}}$ if $L_{v}+L_{\bar{\gamma}}$ is a constant.
}

torus into that region is hindered by the centrifugal barrier which this gas experiences due to its large angular momentum.

Attempts to estimate the characteristic properties of ultrarelativistic outflows (e.g., its terminal Lorentz factor or the collimation factor) on the basis of results from hydrodynamic merger or accretion simulations like the ones presented here must be taken with great caution and might be quantitatively meaningless. The formation of collimated outflows in the vicinity of a torus-accretor system is a violent, time-dependent hydrodynamic process. The jet could clean its own funnel through an initially baryon-loaded environment as happens in the core of a collapsar, where large amounts of gas envelope the black-hole and accretion disk. On the other hand, Kelvin-Helmholtz instabilities due to the interaction of the shear flow with the dense torus may lead to baryon entrainment and collimation. Reliable and quantitatively meaningful answers therefore require the modelling of the jet formation and evolution by hydrodynamic simulations (for more details, see Aloy et al. 2005).

The low-density polar funnels along the rotation axis of the torus are unlikely to form near the poles of a hot, neutrinoradiating massive neutron star, if such a merger remnant escapes the collapse to a black hole. At the surface of such a neutrinoemitting compact object, a dense baryonic wind is driven by neutrino energy deposition. This wind produces an expanding cloud of baryonic matter around the star, a phenomenon which is well-known for the hot proto-neutron stars born in supernova explosions (Duncan et al. 1986; Woosley 1993b; Qian \& Woosley 1996; Thompson et al. 2001).

The hot merger remnant emits neutrinos and antineutrinos with very high luminosities. These energetic neutrinos diffuse and then stream outward to the surface where they must deposit energy by charged-current absorption on free nucleons (the inverse of the reactions Eqs. (8) and (9)) in the layers of decreasing temperature outside of the neutrinosphere. This energy deposition drives the subrelativistic, baryonic wind, whose formation cannot be treated in existing neutron star merger simulations, because the simulations do not include neutrino transport. Neutrino emission is at best described by a neutrino-trapping scheme, in which neutrinos are released locally, but the transport of energy by the neutrinos from layers deep inside the merger remnant to the surface is not followed. Because of the absence of the neutrino-driven wind, the densities in the polar regions of the massive post-merger neutron stars are largely underestimated by the current hydrodynamical models.

The baryon "pollution" by the neutrino-driven wind makes it unlikely that such a hot neutron star merger remnant can be the central engine of a cosmic gamma-ray burst, in particular if the energy release that powers the GRB is considered to happen in the close vicinity of the neutron star. The baryon loading in this region will prevent the formation of a pair-plasma fireball or jet which can accelerate to ultrarelativistic velocities. Neutrino-antineutrino annihilation, for example, deposits energy efficiently only very close to the neutrinosphere and the annihilation rate drops extremely steeply with distance (e.g., like $r^{-8}$ if the neutrino source is a spherical or ellipsoidal body). In case of a hot neutron star the gas density outside of the neutrinosphere is very high - in contrast to the baryon-poor conditions above the poles of a black hole - and therefore $v \bar{v}$ annihilation competes with the charged-current absorption of neutrinos by nucleons (Eqs. (8) and (9)) and with the very efficient cooling due to the inverse of these reactions. In detailed wind models it is therefore seen that $v \bar{v}$ annihilation yields only a minor contribution to the energy transferred to the baryonic wind (see, e.g., Fig. 6 in Thompson et al. 2001), and this additional energy deposition 
triggers enhanced mass loss from the surface of the neutron star instead of driving ultrarelativistic expansion. Similar arguments also apply for models where the GRB outflow is considered to be powered by magnetohydrodynamic activity of a rapidly, differentially rotating hot neutron star. Also in this case it has to be demonstrated that the high baryon densities associated with the neutrino-driven wind around the neutron star do not represent a unsurmountable obstacle for the development of ultrarelativistic fireballs or jets.

\section{Summary}

We have presented 3D hydrodynamic simulations of the timedependent evolution of black-hole accretion-torus systems with parameters typical of the remnants of binary neutron star or neutron-star black-hole mergers. The tori which are created from neutron stars disrupted in such violent events, and the conditions in the torus gas are not well described by steady-state assumptions and the gas evolution requires time-dependent modelling.

The black hole was described by a vacuum boundary condition and pseudo-Newtonian gravitational potential, corresponding to an initial mass of about $4 M_{\odot}$. The effects of black hole rotation were included according to Artemova et al. (1996), taking into account the variation of black hole mass and spin by the accretion of matter from the torus. The torus gas was treated with Newtonian dynamics and self-gravity, its shear viscosity was modelled by a simple $\alpha$-law, and the initial conditions were assumed to be cool (temperatures of 1-2 MeV) and neutron-rich (proton-to-baryon ratio $Y_{\mathrm{e}} \sim 0.02-0.1$ ), decompressed neutron star matter in nuclear statistical equilibrium. The initial spatial distribution of the gas was modelled by using results from (Newtonian) merger simulations.

Assuming different initial masses of the torus $\left(M_{\mathrm{d}, \text { ini }} \sim\right.$ 0.01-0.2 $M_{\odot}$ ), black hole spins (spin parameter $a=0$, $0.3,0.6,0.8)$, and gas viscosity ( $\alpha$-parameter between 0 and 0.1 , we followed the accretion process and the associated neutrino emission for $40 \mathrm{~ms}$ with high-resolution runs, and for $70 \mathrm{~ms}$ with lower resolution runs. The global parameters of both simulations at the same times agree satisfactorily well, but the better resolved simulations exhibit slightly higher gas temperatures and correspondingly larger neutrino emission.

Since our initial conditions with chosen torus mass and black hole potential did not correspond to exact rotational equilibria, the first $10 \mathrm{~ms}$ of our simulations are characterised by a relaxation phase with high mass accretion rates and a dynamical reconfiguration of the torus matter, followed by a more "quiet", quasi-stationary phase in which the mass accretion rate of the black hole settles to a lower, slowly changing value.

The tori start to evolve quickly from their initially neutronrich state to more proton-rich conditions. This protonization proceeds by the emission of electron antineutrinos, which dominates over the emission of electron neutrinos and heavy-lepton neutrinos, and is faster for hotter tori. The gas viscosity thus has an extremely strong influence on the torus evolution. While simulations without physical viscosity $(\alpha=0)$ show only compressional heating and thus stay relatively cool with $Y_{\mathrm{e}}$ changing rather slowly, shear viscosity $(\alpha=0.01$ or 0.1$)$ leads to a rapid rise of the gas temperature, enhanced accretion of the torus regions close to the black hole, and thermally driven inflation of the outer parts of the torus with consequently lower densities. Therefore the mass of viscous tori can decrease initially more rapidly and then continues to decline more slowly with a much reduced mass-loss rate to the black hole. After $40 \mathrm{~ms}$ of evolution the tori have lost about half of their initial mass to the black hole, which at that time accretes matter at rates between about $0.2 M_{\odot} \mathrm{s}^{-1}$ and about $0.6 M_{\odot} \mathrm{s}^{-1}$ in all simulated cases except those with $\alpha=0$ and the highest and lowest initial torus masses.

The maximum densities in the tori have dropped to $\$ 10^{11} \mathrm{~g} \mathrm{~cm}^{-3}$ after $10 \mathrm{~ms}$ in most cases, and stay around $2 \times$ $10^{11} \mathrm{~g} \mathrm{~cm}^{-3}$ after the early relaxation phase only in our most massive accretion tori. The tori are therefore optically thin to neutrinos or develop neutrinospheres just marginally. For this reason neutrinos, once created, stream off essentially freely without being hindered by scattering and reabsorption. Due to the reduced gas densities and relatively high temperatures in viscous tori, the protonization there proceeds quickly and produces $Y_{\mathrm{e}}$-values of $0.3-0.4$ within only $20 \mathrm{~ms}$, tending to establish kinetic equilibrium conditions for the competing $v_{\mathrm{e}}$ and $\bar{v}_{\mathrm{e}}$ emission processes. As a consequence, the $v_{\mathrm{e}}$ and $\bar{v}_{\mathrm{e}}$ luminosities become more similar toward the end of our simulations, although those of electron antineutrinos are still a factor of 2-3 higher. Muon and tau neutrinos and antineutrinos contribute to the energy loss of the accretion tori on a negligible level, because their creation by nucleon-nucleon bremsstrahlung and electron-positron pair annihilation is much slower than the charged-current production of $v_{\mathrm{e}}$ and $\bar{v}_{\mathrm{e}}$ at the temperatures and low densities of the tori.

The effect of the protonization on the neutrino luminosities has interesting consequences for the rate at which neutrinos and antineutrinos deposit energy around the black-hole torus system by the annihilation to electron-positron pairs. While the total neutrino luminosity is strongly correlated with the torus mass and therefore tends to decay with falling gas mass, the energy deposition scales with the product of neutrino and antineutrino luminosities, $L_{v} L_{\bar{v}}$ (see Eq. (12); electron neutrinos and antineutrinos make by far the dominant contribution). It therefore decreases much less steeply with time than the square of the total neutrino luminosities, because the electron neutrino emission is initially very small but rises continuously relative to the one of electron antineutrinos.

As mentioned above, shear viscosity has the largest influence on enhancing the neutrino production. We found the highest luminosities and largest $v \bar{v}$ annihilation rates for our simulations of viscous tori $(\alpha=0.1)$. Values in excess of $10^{53} \mathrm{erg} \mathrm{s}^{-1}$ and up to $\sim 10^{50} \mathrm{erg} \mathrm{s}^{-1}$ for $L_{v}$ and $\dot{E}_{\nu \bar{v}}$, respectively, were reached even for relatively low torus masses on only some $10^{-2} M_{\odot}$. The maximum conversion efficiencies of rest-mass energy to neutrinos could reach $5-10 \%$, and of neutrinos to electron-positron pairs $3-5 \%$. While viscosity, however, tends to decrease the torus lifetime (measured as the ratio of torus mass to mass accretion rate), black hole rotation in the direction of the torus spin has the opposite effect. The higher disk temperatures close to the more compact ISCO in this case, however, have a noticeable effect on the neutrino emission only if the gas viscosity is low or if the black hole spin is close to maximal $(a \gtrsim 0.8)$.

An evaluation of our results for the growth of nonradial instabilities, the presence of which might be suggested by the fact that we found the axial symmetry of our initial models destroyed in a few revolutions, is prevented by our use of a Cartesian grid with three nested levels of different resolution. This grid introduces visible numerical artifacts, in particular imprints a distinct 4-fold symmetry, and must be suspected to be also the reason for higher-order azimuthal modes.

\section{Conclusions and outlook}

Our models are another contribution to the ongoing efforts to theoretically study the viability of accretion tori around 
stellar-mass black holes as central engines of cosmic GRBs. A detailed, critical assessment of previous attempts to link the results of similar simulations to GRB properties has already been made in Sect. 4. The crucial results of our studies in this respect are the neutrino luminosities and the energy conversion by neutrino-antineutrino annihilation, the former being the direct output from the neutrino trapping/leakage scheme applied in our model calculations, the latter being obtained by a postprocessing of our results. A direct calculation of the energy release by $v \bar{v}$-annihilation would require the application of a $3 \mathrm{D}$ transport description, which remains a true challenge for the future.

The results of our studies show that for values of the torus mass, shear viscosity, and black hole rotation well in the range of expectations for compact binary mergers, the neutrino emission is sufficiently powerful and the associated efficiencies for converting gravitational binding energy to neutrinos as well as neutrino-antineutrino pairs to a $\mathrm{e}^{+} \mathrm{e}^{-}$-photon plasma, are sufficiently large to account for the energetics of short GRBs, in particular if collimation of the ultrarelativistic outflow is invoked. Aloy et al. (2005) showed by general relativistic calculations that the fireball-torus interaction can indeed produce collimated jets with "beaming fractions" $f_{\Omega}=2 \delta \Omega_{\text {jet }} /(4 \pi)=1-\cos \theta_{\text {jet }}$ between $\sim 0.5$ and $\sim 2 \%$ of the sky ( $\delta \Omega_{\text {jet }}$ is the solid angle subtended by one jet and $\theta_{\text {jet }}$ its semi-opening angle). This reduces the true energy outputs of the GRBs compared to the observed ones by a factor of 50-200. For the recently detected, short, hard bursts GRB 050509b (Gehrels et al. 2005; Bloom et al. 2006; Hjorth et al. 2005) and GRB 050724 (Berger et al. 2005) this corresponds to true $\gamma$-energies between some $10^{46} \mathrm{ergs}$ and some $10^{48} \mathrm{ergs}$, provided the collimation factor of about 100 inferred by Berger et al. (2005) for GRB 050724 - which is in agreement with the predictions of Aloy et al. (2005) - applies to both bursts. Our results suggest that such energies can be delivered by $v \bar{v}$ annihilation without the need to invoke additional power from mechanisms based on the presence of extremely strong magnetic fields. We therefore refrain from discussing here once more possible ways to use such magnetic fields for tapping the rotational energy of the torus or black hole to drive relativistic motion of small amounts of baryonic matter. We feel unable to provide any deeper insight into such possibilities on grounds of our current simulations than that obtained in the discussions of previous works. These exploit the results of hydrodynamic models to come up with estimates of equilibration field strengths in the swirling gas of the merger remnant, or parametrise the possible energy conversion due to the Blandford-Znajek mechanism in terms of (undetermined or free) system parameters (see, e.g., Rosswog et al. 2003; Rosswog \& Ramirez-Ruiz 2002, 2003; Lee et al. 2005a).

Besides limited numerical resolution, another drawback of 3D simulations is the very limited time over which the evolution of the accretion torus can be followed with the computer resources available to us. The models presented here certainly add only a small brick to our slowly growing picture of the scenario and physics that can make up a GRB engine. The ultimate goal certainly must be simulations which trace the merger history consistently through all phases, from the merging of the compact objects, through the formation of the black hole, to the accretion of the torus matter, until the completion of the phase of powerful energy release from this accretion process. Such simulations will require general relativistic modelling (or a reasonable approximation of it) and should include the microphysics which describes the gas properties as well as the processes that establish the energy loss of the gas and the conversion of some of this energy to relativistic and non-relativistic outflows. Further progress in modelling will not only require adding a spectral treatment of the neutrino emission, preferably by solving the 3D transport problem. It may at some stage also be necessary to perform magnetohydrodynamic simulations for investigating the combined effects of neutrino transport and magnetic fields.

Acknowledgements. We thank the anonymous referee for extensive suggestions which significantly improved and shortened our paper. S.S. highly appreciated continuing encouragement from S. F. Gull and is grateful to G. I. Ogilvie for valuable discussions. S.S. acknowledged support from the Particle Physics and Astronomy Research Council (PPARC), M.R. from the University of Edinburgh Development Trust, H.T.J. from the Sonderforschungsbereich 375 "Astro-Teilchenphysik" und the Sonderforschungsbereich-Transregio 7 "Gravitationswellenastronomie" of the Deutsche Forschungsgemeinschaft. Parts of the simulations were performed at the UK Astrophysical Fluids Facility (UKAFF) and the Edinburgh Parallel Computing Centre (EPCC) of the University of Edinburgh.

\section{References}

Abramowicz, M. A., Chen, X., Kato, S., Lasota, J.-P., \& Regev, O. 1995, ApJ, 438, L37

Aloy, M.A., Janka, H.-T., \& Müller, E. 2005, A\&A, 436, 273

Artemova, I. V., Björnsson, G., \& Novikov, I. D. 1996, ApJ, 461, 565

Balbus, S. A., \& Hawley, J. F. 1998, Rev. Mod. Phys., 70, 1

Berger, E., Price P. A., Cenko S. B., et al, 2005, Nature 438, 988.

Blandford, R. D., \& Znajek, R. L. 1977, MNRAS, 179, 433

Blinnikov, S. I., Novikov, I. D., Perevodchikova, T. V., \& Polnarev, A. G. 1984, Pis'ma Astron. Zh., 10, 422

Bloom, J. S., Prochaska, J. X., Pooley, D., et al. 2006, ApJ, 638, 354

Chen, X., Abramowicz, M. A., Lasota, J.-P., Narayan, R., \& Yi, I. 1995, ApJ, 443, L61

Colella, P., \& Woodward, P. R. 1984, JCP, 54, 174

Di Matteo, T., Perna, R., \& Narayan, R. 2002, ApJ, 579, 706

Duez, M. D., Liu, Y. T., Shapiro, S. L., \& Stephens, B. C., Phys. Rev. D, 69, 4030

Duncan, R. C., Shapiro, S. L., \& Wasserman, I., ApJ, 309, 141

Eichler, D., Livio, M., Piran, T., \& Schramm, D. N. 1989, Nature, 340, 126

Gehrels, N., Barbier L., Barthelmy S. D., et al, 2005, Nature, [arXiv: astro-ph/0505630]

Goodman, J. 1986, ApJ, 308, L47

Goodman, J., Dar A., \& Nussinov, S. 1987, ApJ, 314, L7

Hawley, J. F., \& Krolik, J. H. 2001, ApJ, 548, 348

Hjorth, J., Sollerman, J., Gorosabel, J., et al. 2005, ApJ, 630, L117

Janka, H.-Th., \& Ruffert, M. 1996, A\&A, 307, L33

Janka, H.-Th., Eberl, T., Ruffert, M., \& Fryer, C. L. 1999, ApJ, 527, L39

Jaroszyński, M. 1993, Acta Astron., 43, 183

Jaroszyński, M. 1996, A\&A, 305, 839

Kleiber, R., \& Glatzel, W. 1999, MNRAS, 303, 107

Kluźniak, W., \& Lee, W. H. 1998, ApJ, 494, L53

Kohri, K., \& Mineshige, S. 2002, ApJ, 577, 311

Kulkarni, S. R., Djorgovski, S. G., Ramagrakash, A. N., et al. 1998, Nature, 393, 35

Lattimer, J. M., \& Swesty, F. D. 1991, Nucl. Phys. A, 535, 331

Lee, H. K., Wijers, R. A. M. J., \& Brown, G. E. 2000, Phys. Rep., 325, 83

Lee, W. H., \& Kluźniak, W. 1995, Acta Astron., 45, 705

Lee, W. H., \& Kluźniak, W. 1998, ApJ, 526, 178

Lee, W. H., \& Ramirez-Ruiz, E. 2002, ApJ, 577, 893

Lee, W. H., Ramirez-Ruiz, E., \& Page, D. 2004, ApJ, 608, L5

Lee, W. H., Ramirez-Ruiz, E., \& Page, D. 2005a, ApJ, 632, 421

Lee, W. H., Ramirez-Ruiz, E., \& Granot, J. 2005b, ApJ, 630, L165

Li, L.-X. 2000, ApJ, 533, L115

Livio, M., Ogilvie, G. I., \& Pringle, J. E. 1999, ApJ, 512, 100

MacFadyen, A., \& Woosley, S. E. 1999, ApJ, 524, 262

McKinney, J. C., \& Gammie, C. F. 2002, ApJ, 573, 728

Mészáros, P., \& Rees, M. J. 1992, ApJ, 397, 570

Mészáros, P., \& Rees, M. J. 1993, ApJ, 405, 278

Mészáros, P., \& Rees, M. J. 1997, ApJ, 482, L29

Mészáros, P., Rees, M. J., \& Wijers, R. A. M. J. 1999, New Astron., 4, 303

Mochkovitch, R., Hernanz, M., Isern, J., \& Martin, X. 1993, Nature, 361, 236

Mochkovitch, R., Heranz, M., Isern, J., \& Loiseau, S. 1995, A\&A, 293, 803

Morrison, I. A., Baumgarte, T. W., \& Shapiro, S. L. 2004, ApJ, 610, 941

Narayan, R., Paczyński, B., \& Piran, T. 1992, ApJ, 395, L83

Narayan, R., Piran, T., \& Kumar, P. 2001, ApJ, 557, 949

Oechslin, R., \& Janka, H.-Th. 2006, MNRAS, 368, 1489 
Oechslin, R., Uryū, K., Poghosyan, G., \& Thielemann, F.-K. 2004, MNRAS, 349, 1469

Paczyński, B., \& Wiita, P. J. 1980, A\&A, 88, 23

Paczyński, B. 1986, ApJ, 308, L4

Paczyński, B. 1991, Acta Astron., 41, 257

Papaloizou, J. C. B., \& Pringle, J. E. 1984, MNRAS, 208, 721

Popham, R., Woosley, S. E., \& Fryer, C. 1999, ApJ, 518, 356

Qian, Y.-Z., \& Woosley, S. E., ApJ, 471, 331

Rosswog, S., Davies, M. B., Thielemann, F.-K., \& Piran, T., 2000, A\&A, 360, 171

Rosswog, S., Liebendörfer, M., Thielemann, F.-K., et al. 1999, A\&A, 341, 499

Rosswog, S., \& Liebendörfer, M. 2003, MNRAS, 342, 673

Rosswog, S., \& Ramirez-Ruiz, E. 2002, MNRAS, 336, L7

Rosswog, S., \& Ramirez-Ruiz, E. 2003, MNRAS, 343, L36

Rosswog, S., Ramirez-Ruiz, E., \& Davies, M. B., 2003, MNRAS, 345, 1077
Rosswog, S., Speith, R., \& Wynn, G. A., 2004, MNRAS, 351, 1121 Ruffert, M. 1992, A\&A, 265, 82

Ruffert, M., \& Janka, H.-Th. 1999, A\&A, 344, 573

Ruffert, M., \& Janka, H.-Th. 2001, A\&A, 380, 544

Ruffert, M., Janka, H.-Th., \& Schäfer, G. 1996, A\&A, 311, 532

Ruffert, M., Janka, H.-Th., Takahashi, K., \& Schäfer, G. 1997, A\&A, 319, 122

Setiawan, S., Ruffert, M., \& Janka, H.-Th. 2004, MNRAS, 352, 753

Shakura, N. I., \& Sunyaev, R. A. 1973, A\&A, 24, 337

Thompson, C. 1994, MNRAS, 270, 480

Thompson, T. A., Burrows, A., \& Meyer, B. S. 2001, ApJ, 562, 887

Thompson, T. A., Quarteart, E., \& Burrows, A. 2005, ApJ, 620, 861

Witt, H. J., Jaroszyński, M., Haensel, P., Paczyński, B., \& Wambsganss, J. 1994, ApJ, 422, 219

Woosley, S. E. 1993a, ApJ, 405, 273

Woosley, S. E. 1993b, A\&AS, 97, 205 